\title{
Ancient human genomes suggest three ancestral populations for present-day Europeans
}

\author{
A list of authors and their affiliations appears at the end of the paper
}

\begin{abstract}
We sequenced the genomes of a $\sim 7,000$-year-old farmer from Germany and eight $\sim 8,000$-year-old hunter-gatherers from Luxembourg and Sweden. We analysed these and other ancient genomes ${ }^{1-4}$ with 2,345 contemporary humans to show that most present-day Europeans derive from at least three highly differentiated populations: west European hunter-gatherers, who contributed ancestry to all Europeans but not to Near Easterners; ancient north Eurasians related to Upper Palaeolithic Siberians ${ }^{3}$, who contributed to both Europeans and Near Easterners; and early European farmers, who were mainly of Near Eastern origin but also harboured west European hunter-gatherer related ancestry. We model these populations' deep relationships and show that early European farmers had $\sim \mathbf{4 4 \%}$ ancestry from a 'basal Eurasian' population that split before the diversification of other non-African lineages.
\end{abstract}

Near Eastern migrants from Anatolia and the Levant are known to have played a major role in the introduction of agriculture to Europe, as ancient DNA indicates that early European farmers were distinct from European hunter-gatherers ${ }^{4,5}$ and close to present-day Near Easterners ${ }^{4,6}$. However, modelling present-day Europeans as a mixture of these two ancestral populations ${ }^{4}$ does not account for the fact that Europeans are also admixed with a population related to Native Americans ${ }^{7,8}$. To clarify the prehistory of Europe, we sequenced nine ancient genomes (Fig. 1 and Extended Data Fig. 1): 'Stuttgart' (19-fold coverage), a 7,000-yearold skeleton found in Germany in the context of artefacts from the first widespread farming culture of central Europe, the Linearbandkeramik; 'Loschbour' (22-fold), an 8,000-year-old skeleton from the Loschbour rock shelter in Luxembourg, discovered in the context of hunter-gatherer artefacts (Supplementary Information sections 1 and 2); and seven $\sim 8,000$ year-old samples (0.01-2.4-fold) from a hunter-gatherer burial in Motala, Sweden (the highest coverage individual was 'Motala12').

Sequence reads from all samples revealed $>20 \% \mathrm{C} \rightarrow \mathrm{T}$ and $\mathrm{G} \rightarrow \mathrm{A}$ deamination-derived mismatches at the ends of the molecules that are characteristic of ancient $\mathrm{DNA}^{9,10}$ (Supplementary Information section 3). We estimate nuclear contamination rates to be $0.3 \%$ for Stuttgart and $0.4 \%$ for Loschbour (Supplementary Information section 3 ), and mitochondrial (mtDNA) contamination rates to be $0.3 \%$ for Stuttgart, $0.4 \%$ for Loschbour, and $0.01-5 \%$ for the Motala individuals (Supplementary Information section 3). Stuttgart has mtDNA haplogroup T2, typical of Neolithic Europeans ${ }^{11}$, and Loschbour and all Motala individuals have the U5 or U2 haplogroups, typical of hunter-gatherers ${ }^{5,9}$ (Supplementary Information section 4). Stuttgart is female, whereas Loschbour and five Motala individuals are male (Supplementary Information section 5) and belong to Y-chromosome haplogroup I, suggesting that this was common in pre-agricultural Europeans (Supplementary Information section 5).

We carried out large-scale sequencing of libraries prepared with uracil DNA glycosylase (UDG), which removes deaminated cytosines, thus reducing errors arising from ancient DNA damage (Supplementary Information section 3). The ancient individuals had indistinguishable levels of Neanderthal ancestry when compared to each other $(\sim 2 \%)$ and to present-day Eurasians (Supplementary Information section 6). The heterozygosity of Stuttgart (0.00074) is at the high end of present-day Europeans, whereas that of Loschbour (0.00048) is lower than in any present human populations (Supplementary Information section 2); this must reflect a strong bottleneck in Loschbour's ancestors, as the genetic data show that he was not recently inbred (Extended Data Fig. 2). High copy numbers for the salivary amylase gene (AMY1) have been associated with a high starch $\operatorname{diet}^{12}$; our ancient genomes are consistent with the direction of this observation in that the Stuttgart farmer had the highest number of copies (16), whereas the ancient hunter-gatherers La Braña (from Iberia) $)^{2}$, Motala12, and Loschbour had lower numbers (5, 6 and 13, respectively) (Supplementary Information section 7). We caution, however, that copy count in Loschbour is at the high end of present-day humans, showing that high copy counts of AMY1 cannot be accounted for entirely by selection since the switch to agriculture. Both Loschbour and Stuttgart had dark hair ( $>99 \%$ probability); and Loschbour, like La Braña and Motala12, probably had blue or light coloured eyes $(>75 \%)$ whereas Stuttgart probably had brown eyes ( $>99 \%$ probability) (Supplementary Information section 8). Neither Loschbour nor La Braña carries the skin-lightening allele in SLC24A5 that is homozygous in Stuttgart and nearly fixed in Europeans today ${ }^{2}$, but Motala12 carries at least one copy of the derived allele, showing that this allele was present in Europe before the advent of agriculture.

We compared the ancient genomes to 2,345 present-day humans from 203 populations genotyped at 594,924 autosomal single nucleotide polymorphisms (SNPs) with the Human Origins array ${ }^{8}$ (Supplementary Information section 9) (Extended Data Table 1). We used ADMIXTURE ${ }^{13}$ to identify 59 'west Eurasian' populations that cluster with Europe and the Near East (Supplementary Information section 9 and Extended Data Fig. 3). Principal component analysis (PCA $)^{14}$ (Supplementary Information section 10) (Fig. 2) indicates a discontinuity between the Near East and Europe, with each showing north-south clines bridged only by a few populations of mainly Mediterranean origin. We projected ${ }^{15}$ the newly sequenced and previously published ${ }^{1-4}$ ancient genomes onto the first two principal components (PCs) (Fig. 2). Upper Palaeolithic huntergatherers $^{3}$ from Siberia like the MA1 (Mal'ta) individual project at the northern end of the PCA, suggesting an 'ancient north Eurasian' (ANE) meta-population. European hunter-gatherers from $\mathrm{Spain}^{2}$, Luxembourg, and Sweden ${ }^{4}$ fall beyond present-day Europeans in the direction of European differentiation from the Near East, and form a 'west European hunter-gatherer' (WHG) cluster including Loschbour and La Braña ${ }^{2}$, and a 'Scandinavian hunter-gatherer' (SHG) cluster including the Motala individuals and $\sim 5,000$-year-old hunter-gatherers from the Pitted Ware Culture 4 . An 'early European farmer' (EEF) cluster includes Stuttgart, the $\sim 5,300$-year-old Tyrolean Iceman ${ }^{1}$ and a $\sim 5,000$-year-old Swedish farmer ${ }^{4}$.

Patterns observed in PCA may be affected by sample composition (Supplementary Information section 10) and their interpretation in terms of admixture events is not straightforward, so we rely on formal analysis of $f$ statistics $^{8}$ to document mixture of at least three source populations in the ancestry of present Europeans. We began by computing all possible statistics of the form $f_{3}\left(\right.$ Test; $R e f_{1}, \operatorname{Ref}_{2}$ ) (Supplementary Information section 11), which if significantly negative show unambiguously ${ }^{8}$ that Test is admixed between populations anciently related to $R e f_{1}$ and $R e f_{2}$ (we choose $R e f_{1}$ and $R e f_{2}$ from 5 ancient and 192 present populations). The lowest $f_{3}$ statistics for Europeans are negative (93\% are $>4$ standard errors below 0 ), with most showing strong support for at least one 


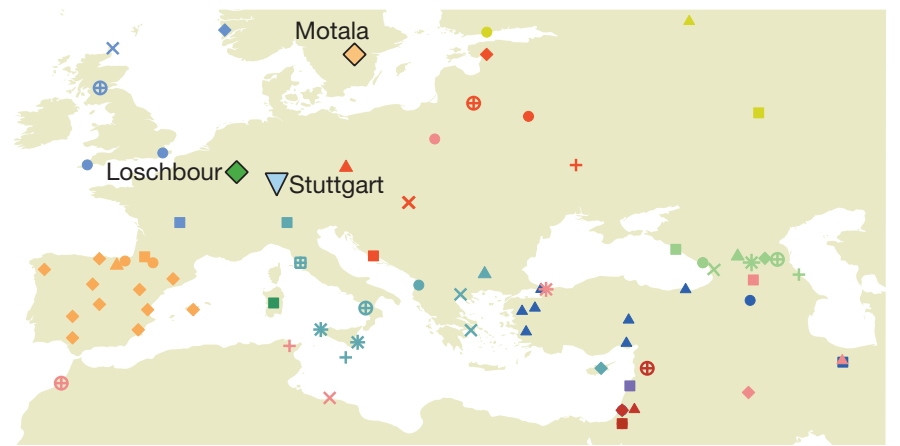

Figure 1 Map of west Eurasian populations. Geographical locations of analysed samples, with colour coding matching the PCA (Fig. 2). We show all sampling locations for each population, which results in multiple points for some (for example, Spain).

ancient individual being one of the references (Supplementary Information section 11). Europeans almost always have their lowest $f_{3}$ with either (EEF, ANE) or (WHG, Near East) (Supplementary Information section 11, Table 1 and Extended Data Table 1), which would not be expected if there were just two ancient sources of ancestry (in which case the best references for all Europeans would be similar). The lowest $f_{3}$ statistic for Near Easterners always takes Stuttgart as one of the reference populations, consistent with a Near Eastern origin for Stuttgart's ancestors (Table 1). We also computed the statistic $f_{4}$ (Test, Stuttgart; MA1, Chimp), which measures whether MA1 shares more alleles with a Test population or with Stuttgart. This statistic is significantly positive (Extended Data Fig. 4 and Extended Data Table 1) if Test is nearly any present-day West Eurasian population, showing that MA1-related ancestry has increased since the time of early farmers like Stuttgart (the same statistic using Native Americans instead of MA1 has the same sign but is smaller in magnitude (Extended Data Fig. 5), indicating that MA1 is a better surrogate than the Native Americans who were first used to document ANE ancestry in Europe $\left.{ }^{7,8}\right)$. The analogous statistic $f_{4}($ Test, Stuttgart; Loschbour, Chimp) is nearly always positive in Europeans and negative in Near Easterners, indicating that Europeans have more ancestry from populations related to Loschbour than do Near Easterners (Extended Data Fig. 4 and Extended Data Table 1). Extended Data Table 2 documents the robustness of key $f_{4}$ statistics by recomputing them using transversion polymorphisms not affected by ancient DNA damage, and also using whole-genome sequencing data not affected by SNP ascertainment bias. Extended Data Fig. 6 shows the geographic gradients in the degree of allele sharing of present-day West Eurasians (as measured by $f_{4}$ statistics) with Stuttgart (EEF), Loschbour (WHG) and MA1 (ANE).

To determine the minimum number of source populations needed to explain the data for many European populations taken together, we studied the matrix of all possible statistics of the form $f_{4}\left(\right.$ Test $_{\text {base }}$ Test $t_{\mathrm{i}}$; $O_{\text {base }} O_{\mathrm{j}}$ ) (Supplementary Information section 12). Test $t_{\text {base }}$ is a reference European population, Test $t_{\mathrm{i}}$ is the set of all other European Test populations, $O_{\text {base }}$ is a reference outgroup, and $O_{\mathrm{j}}$ is the set of other outgroups (ancient DNA samples, Onge, Karitiana, and Mbuti). The rank of the $(i, j)$ matrix reflects the minimum number of sources that contributed to the Test populations ${ }^{16,17}$. For a pool of individuals from 23 Test populations representing most present-day European groups, this analysis rejects descent from just two sources $\left(P<10^{-12}\right.$ by a Hotelling $t$-test $\left.{ }^{17}\right)$. However, three source populations are consistent with the data after excluding the Spanish who have evidence for African admixture ${ }^{18-20}(P=0.019$, not significant after multiple-hypothesis correction), consistent with the results from ADMIXTURE (Supplementary Information section 9), PCA (Fig. 2 and Supplementary Information section 10) and $f$ statistics (Extended Data Table 1, Extended Data Fig. 6, Supplementary Information sections 11 and 12). We caution that the finding of three sources could be consistent with a larger number of mixture events. Moreover, the source

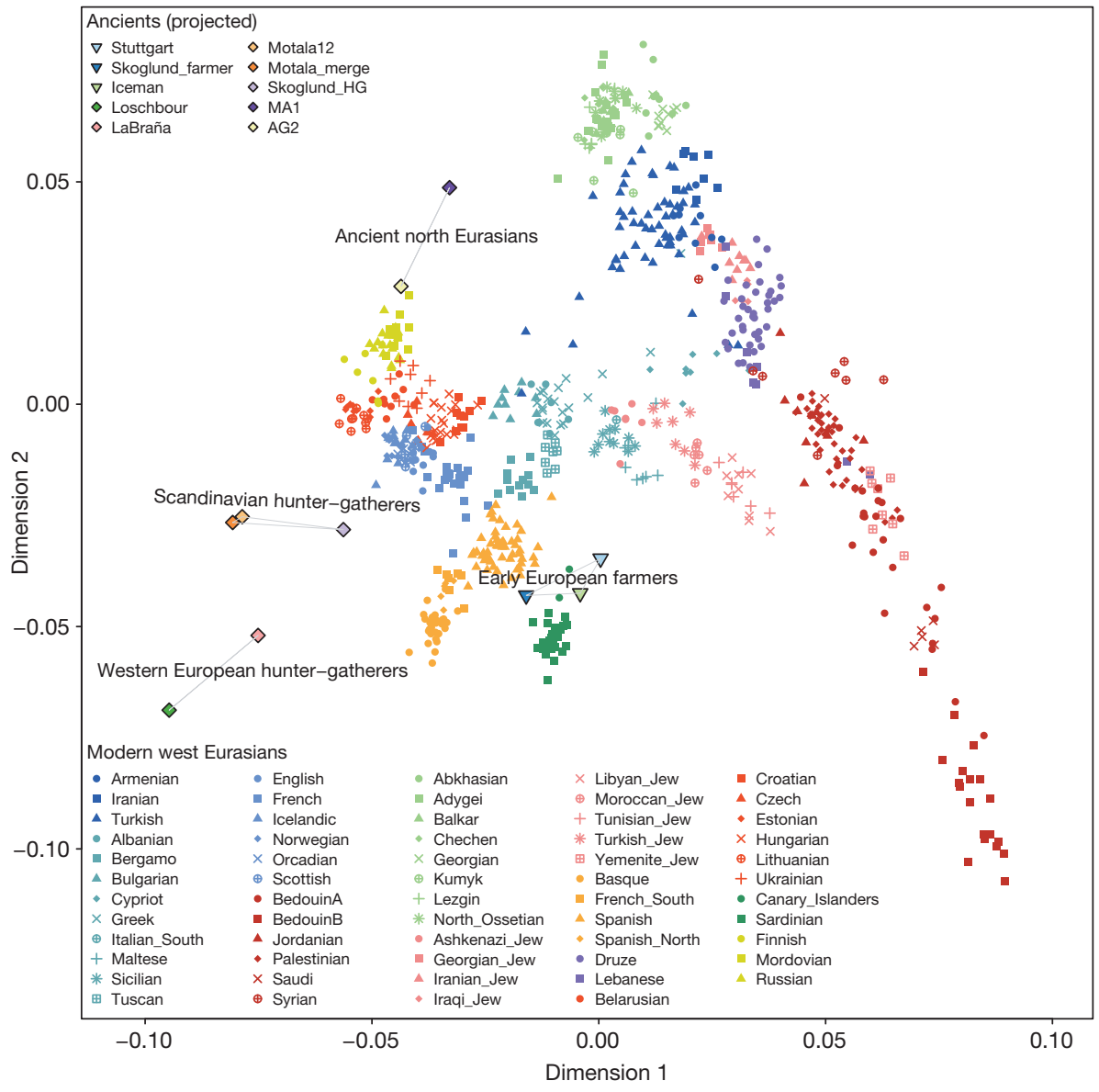

Figure $2 \mid$ Principal Component Analysis. PCA on all present-day west Eurasians, with ancient samples projected. European huntergatherers fall beyond present-day Europeans in the direction of European differentiation from the Near East. Stuttgart clusters with other Neolithic Europeans and present-day Sardinians. MA1 falls outside the variation of present-day west Eurasians in the direction of southern-northern differentiation along dimension 2 . 
Table 1 | Lowest $f_{3}$ statistics for each west Eurasian population

\begin{tabular}{|c|c|c|}
\hline $\operatorname{Ref}_{1}$ & $\operatorname{Ref}_{2}$ & Target for which these two references give the lowest $f_{3}\left(X ; \operatorname{Ref}_{1}, \operatorname{Ref}_{2}\right)$ \\
\hline WHG & EEF & Sardinian*** \\
\hline WHG & Near East & $\begin{array}{l}\text { Basque, Belarusian, Czech, English, Estonian, Finnish, French_South, Icelandic, Lithuanian, Mordovian, Norwegian, Orcadian, } \\
\text { Scottish, Spanish, Spanish_North, Ukrainian }\end{array}$ \\
\hline WHG & Siberian & Russian \\
\hline EEF & ANE & $\begin{array}{l}\text { Abkhasian***, Albanian, Ashkenazi_Jew****, Bergamo, Bulgarian, Chechen****, Croatian, Cypriot****, Druze**, French, Greek, } \\
\text { Hungarian, Lezgin, Maltese, Sicilian, Turkish_Jew, Tuscan }\end{array}$ \\
\hline EEF & Native American & Adygei, Balkar, Iranian, Kumyk, North_Ossetian, Turkish \\
\hline EEF & African & $\begin{array}{l}\text { BedouinA, BedouinB†, Jordanian, Lebanese, Libyan_Jew, Moroccan_Jew, Palestinian, Saudi****, Syrian, Tunisian_Jew***, } \\
\text { Yemenite_Jew*** }\end{array}$ \\
\hline EEF & South Asian & Armenian, Georgian****, Georgian_Jew*, Iranian_Jew***, Iraqi_Jew*** \\
\hline
\end{tabular}

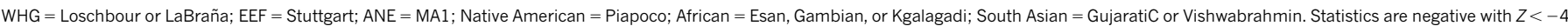
unless otherwise noted: $\dagger$ (positive) or *,**,***,****, to indicate $Z$ less than $0,-1,-2$, and -3 , respectively. The complete list of statistics can be found in Extended Data Table 1 .

populations may themselves have been mixed. Indeed, the positive $f_{4}$ (Stuttgart, Test; Loschbour, Chimp) statistics obtained when Test is Near Eastern (Extended Data Table 1) imply that the EEF had some WHGrelated ancestry, which was greater than $0 \%$ and as high as $45 \%$ (Supplementary Information section 13).

We used the ADMIXTUREGRAPH software ${ }^{8,15}$ to fit a model (a tree structure augmented by admixture events) to the data, exploring models relating the three ancient populations (Stuttgart, Loschbour, and MA1) to two eastern non-Africans (Onge and Karitiana) and sub-Saharan Africans (Mbuti). We found no models that fit the data with 0 or 1 admixture events, but did find a model that fit with 2 admixture events (Supplementary Information section 14). The successful model (Fig. 3) confirms the existence of MA1-related admixture in Native Americans ${ }^{3}$, but includes the novel inference that Stuttgart is partially ( $44 \pm 10 \%)$ derived from a lineage that split before the separation of eastern non-Africans from the common ancestor of WHG and ANE. The existence of such basal Eurasian admixture into Stuttgart provides a simple explanation for our finding that diverse eastern non-African populations share significantly more alleles with ancient European and Upper Palaeolithic Siberian

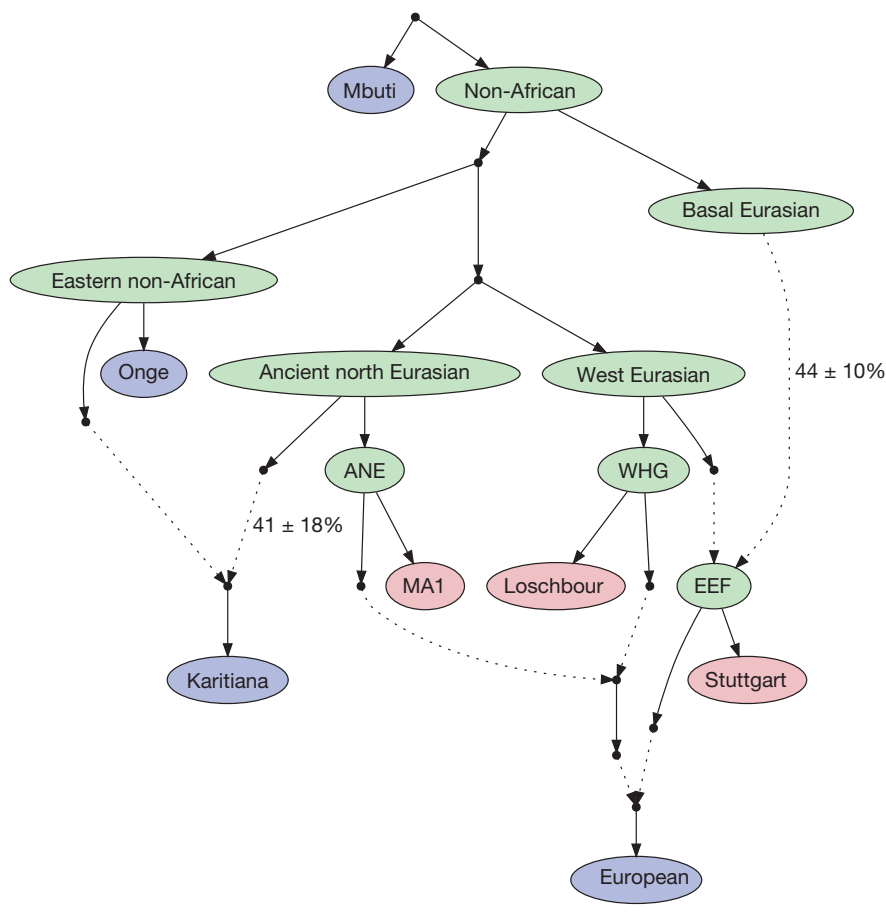

Figure $3 \mid$ Modelling the relationship of European to non-European populations. A three-way mixture model that is a fit to the data for many populations. Present-day samples are coloured in blue, ancient in red, and reconstructed ancestral populations in green. Solid lines represent descent without mixture, and dashed lines represent admixture. We print mixture proportions and one standard error for the two mixtures relating the highly divergent ancestral populations. (We do not print the estimate for the 'European' population as it varies depending on the population.) hunter-gatherers than with Stuttgart (that is, $f_{4}($ Eastern non-African, Chimp; Hunter-gatherer, Stuttgart) is significantly positive), but that hunter-gatherers appear to be equally related to most eastern groups (Supplementary Information section 14). We verified the robustness of the model by reanalysing the data using the unsupervised MixMapper ${ }^{7}$ (Supplementary Information section 15) and TreeMix ${ }^{21}$ software (Supplementary Information section 16), which both identified the same admixture events. The ANE-WHG split must have occurred $>24,000$ years ago (as it must predate the age of MA1 (ref. 3)), and the WHG and Eastern non-African split must have occurred $>40,000$ years ago (as it must predate the Tianyuan ${ }^{22}$ individual from China which clusters with Asians to the exclusion of Europeans). The basal Eurasian split must be even older, and might be related to early settlement of the Levant ${ }^{23}$ or Arabia ${ }^{24,25}$ before the diversification of most Eurasians, or more recent gene flow from Africa ${ }^{26}$. However, the basal Eurasian population shares much of the genetic drift common to non-African populations after their separation from Africans, and thus does not appear to represent gene flow between sub-Saharan Africans and the ancestors of non-Africans after the out-ofAfrica bottleneck (Supplementary Information section 14).

Fitting present-day Europeans into the model, we find that few populations can be fit as two-way mixtures, but nearly all are compatible with three-way mixtures of ANE-EEF-WHG (Supplementary Information section 14). The mixture proportions from the fitted model (Fig. 4 and Extended Data Table 3) are encouragingly consistent with those obtained from a separate method that relates European populations to diverse outgroups using $f_{4}$ statistics, assuming only that MA1 is an unmixed descendent of ANE, Loschbour of WHG, and Stuttgart of EEF (Supplementary Information section 17). We infer that EEF ancestry in Europe today ranges from $\sim 30 \%$ in the Baltic region to $\sim 90 \%$ in the Mediterranean, consistent with patterns of identity-by-descent (IBD) sharing ${ }^{27,28}$ (Supplementary Information section 18) and shared haplotype analysis (chromosome painting $)^{29}$ (Supplementary Information section 19) in which Loschbour shares more segments with northern Europeans and Stuttgart with southern Europeans. Southern Europeans inherited their European hunter-gatherer ancestry mostly via EEF ancestors (Extended Data Fig. 6), whereas northern Europeans acquired up to $50 \%$ of WHG ancestry above and beyond what they received through their EEF ancestors. Europeans have a larger proportion of WHG than ANE ancestry in general. By contrast, in the Near East there is no detectable WHG ancestry, but up to $\sim 29 \%$ ANE in the Caucasus (Supplementary Information section 14). A striking feature of these findings is that ANE ancestry is inferred to be present in nearly all Europeans today (with a maximum of $\sim 20 \%$ ), but was absent in both farmers and hunter-gatherers from central and western Europe during the Neolithic transition. However, ANE ancestry was not completely absent from the larger European region at that time: we find that it was present in $\sim 8,000$-years-old Scandinavian hunter-gatherers, as MA1 shares more alleles with Motala12 (SHG) than with Loschbour, and Motala12 fits as a mixture of $81 \%$ WHG and 19\% ANE (Supplementary Information section 14).

Two sets of European populations are poor fits for the model. Sicilians, Maltese, and Ashkenazi Jews have EEF estimates of $>100 \%$, consistent with their having more Near Eastern ancestry than can be explained via 


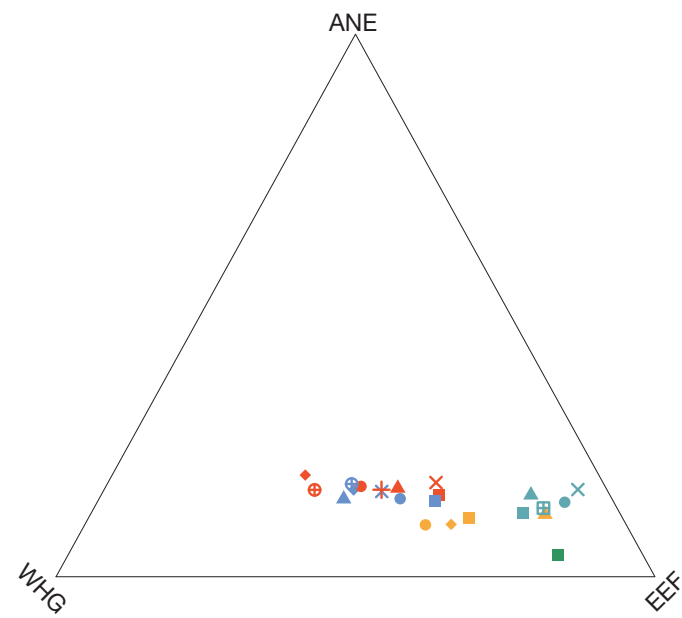
- Albanian
- French
- Bergamo
- Icelandic
- French_South
- Spanish
- Spanish_North
- Estonian
- Bulgarian
- Norwegian
- Belarusian
$\times$ Orcadian
- Croatian
- Czech
$\times$ Hungarian
$\oplus$ Lithuanian
+ Ukrainian
t Tuscan
- English
- Basque
- Sardinian

Figure $4 \mid$ Estimates of mixture proportions in present-day Europeans. Plot of the proportions of ancestry from each of three inferred ancestral populations (EEF, ANE and WHG).

EEF admixture (Supplementary Information section 17). They also cannot be jointly fit with other Europeans (Supplementary Information section 14), and they fall in the gap between European and Near Easterners in PCA (Fig. 2). Finns, Mordovians and Russians (from the northwest of Russia) also do not fit (Supplementary Information section 14; Extended Data Table 3) due to East Eurasian gene flow into the ancestors of these north-eastern European populations. These populations (and Chuvash and Saami) are more related to east Asians than can be explained by ANE admixture (Extended Data Fig. 7), probably reflecting a separate stream of Siberian gene flow into north-eastern Europe (Supplementary Information section 14).

Several questions will be important to address in future ancient DNA work. One question concerns where and when the Near Eastern farmers mixed with European hunter-gatherers to produce the EEF. A second question concerns how the ancestors of present-day Europeans first acquired their ANE ancestry. Discontinuity in central Europe during the late Neolithic ( $\sim 4,500$ years ago) associated with the appearance of mtDNA types absent in earlier farmers and hunter-gatherers ${ }^{30}$ raises the possibility that ANE ancestry may have also appeared at this time. Finally, it will be important to study ancient genome sequences from the Near East to provide insights into the history of the basal Eurasians.

Online Content Methods, along with any additional Extended Data display items and Source Data, are available in the online version of the paper; references unique to these sections appear only in the online paper.

\section{Received 23 December 2013; accepted 11 July 2014}

1. Keller, A. et al. New insights into the Tyrolean Iceman's origin and phenotype as inferred by whole-genome sequencing. Nature Commun. 3, 698 (2012)

2. Olalde, I. et al. Derived immune and ancestral pigmentation alleles in a 7,000-yearold Mesolithic European. Nature 507, 225-228 (2014).

3. Raghavan, M. et al. Upper Palaeolithic Siberian genome reveals dual ancestry of Native Americans. Nature 505, 87-91 (2014).

4. Skoglund, P. et al. Origins and genetic legacy of Neolithic farmers and huntergatherers in Europe. Science 336, 466-469 (2012).

5. Bramanti, B. et al. Genetic discontinuity between local hunter-gatherers and Central Europe's first farmers. Science 326, 137-140 (2009).

6. Haak, W. et al. Ancient DNA from European early Neolithic farmers reveals their Near Eastern affinities. PLoS Biol. 8, e1000536 (2010)

7. Lipson, M. et al. Efficient moment-based inference of admixture parameters and sources of gene flow. Mol. Biol. Evol. 30, 1788-1802 (2013).

8. Patterson, N. et al. Ancient admixture in human history. Genetics 192, 1065-1093 (2012).

9. Krause, J et al. A complete mtDNA genome of an early modern human from Kostenki, Russia. Curr. Biol. 20, 231-236 (2010).

10. Sawyer, S., Krause, J., Guschanski, K., Savolainen, V.\& Pääbo, S. Temporal patterns of nucleotide misincorporations and DNA fragmentation in ancient DNA. PLoS ONE 7, e34131 (2012)

11. Haak, W. et al. Ancient DNA from the first European farmers in 7500-year-old Neolithic sites. Science 310, 1016-1018 (2005).

12. Perry, G. H. et al. Diet and the evolution of human amylase gene copy number variation. Nature Genet. 39, 1256-1260 (2007).

13. Alexander, D. H., Novembre, J. \& Lange, K. Fast model-based estimation of ancestry in unrelated individuals. Genome Res. 19, 1655-1664 (2009).

14. Patterson, N., Price, A. L. \& Reich, D. Population structure and eigenanalysis. PLoS Genet. 2, e190 (2006)

15. Reich, D., Thangaraj, K., Patterson, N., Price, A. L. \& Singh, L. Reconstructing Indian population history. Nature 461, 489-494 (2009).

16. Moorjani, P. et al. Genetic evidence for recent population mixture in India. Am J. Hum. Genet. 93, 422-438 (2013).

17. Reich, D. et al. Reconstructing Native American population history. Nature 488, 370-374 (2012)

18. Botigué, L. R. et al. Gene flow from North Africa contributes to differential human genetic diversity in southern Europe. Proc. Natl Acad. Sci. USA 110, 11791-11796 (2013)

19. Cerezo, M. et al. Reconstructing ancient mitochondrial DNA links between Africa and Europe. Genome Res. 22, 821-826 (2012).

20. Moorjani, P. et al. The history of African gene flow into southern Europeans, Levantines, and Jews. PLoS Genet. 7, e1001373 (2011).

21. Pickrell, J. K. \& Pritchard, J. K. Inference of population splits and mixtures from genome-wide allele frequency data. PLoS Genet. 8, e1002967 (2012).

22. Fu, Q. et al. DNA analysis of an early modern human from Tianyuan Cave, China Proc. Natl Acad. Sci. USA 110, 2223-2227 (2013).

23. Bar-Yosef, O. The Chronology of the Middle Paleolithic of the Levant 39-56 (Plenum Press, 1998)

24. Armitage, S. J. et al. The southern route "out of Africa": evidence for an early expansion of modern humans into Arabia. Science 331, 453-456 (2011).

25. Rose, J. I. et al. The Nubian Complex of Dhofar, Oman: an African middle stone age industry in Southern Arabia. PLoS ONE 6, e28239 (2011).

26. Brace, C. L. et al. The questionable contribution of the Neolithic and the Bronze Age to European craniofacial form. Proc. Natl Acad. Sci. USA 103, 242-247 (2006)

27. Browning, B. L. \& Browning, S. R. Improving the accuracy and efficiency of identity-by-descent detection in population data. Genetics 194, 459-471 (2013).

28. Ralph, P.\& Coop, G. The geography of recent genetic ancestry across Europe. PLoS Biol. 11, e1001555 (2013).

29. Lawson, D. J., Hellenthal, G., Myers, S. \& Falush, D. Inference of population structure using dense haplotype data. PLoS Genet. 8, e1002453 (2012).

30. Brandt, G. et al. Ancient DNA reveals key stages in the formation of central European mitochondrial genetic diversity. Science 342, 257-261 (2013)

Supplementary Information is available in the online version of the paper.

Acknowledgements We thank the 1,615 volunteers from 147 diverse populations who donated DNA samples and whose genetic data are newly reported in this study. We are grateful to C. Beall, N. Bradman, A. Gebremedhin, D. Labuda, M. Nelis and A. Di Rienzo for sharing DNA samples; to D. Weigel, C. Lanz, V. Schünemann, P. Bauer and O. Riess for support and access to DNA sequencing facilities; to $P$. Johnson for advice on contamination estimation; to G. Hellenthal for help with the ChromoPainter software; and to P. Skoglund for sharing graphics software. We thank K. Nordtvedt for alerting us to newly discovered Y-chromosome SNPs. We downloaded the POPRES data from $\mathrm{dbGaP}$ at (http://www.ncbi.nlm.nih.gov/projects/gap/cgi-bin/study.cgi?study $\mathrm{id}=$ phs000145.v4.p2) through dbGaP accession number phs000145.v1.p2. We thank all the volunteers who donated DNA. We thank the staff of the Unità Operativa Complessa di Medicina Trasfusionale, Azienda Ospedaliera Umberto I, Siracusa, Italy for assistance in sample collection; and The National Laboratory for the Genetics of Israeli Populations for facilitating access to DNA. We thank colleagues at the Applied Genomics at the Children's Hospital of Philadelphia, especially H. Hakonarson, C. Kim, K. Thomas, and C. Hou, for genotyping samples on the Human Origins array. J.Kr., A.M. and C.P. are grateful for support from DFG grant number KR 4015/1-1, the Carl-Zeiss Foundation and the Baden Württemberg Foundation. S.P., G.R., Q.F., C.F., K.P., S.C. and J.Ke. acknowledge support from the Presidential Innovation Fund of the Max Planck Society. G.R. was supported by an NSERC fellowship. J.G.S. acknowledges use of the Extreme Science and Engineering Discovery Environment (XSEDE), which is supported by NSF grant number OCl-1053575. E.B. and O.B. were supported by RFBR grants 13-06-00670, 13-04-01711, 13-04-90420 and by the Molecular and Cell Biology Program of the Presidium, Russian Academy of Sciences. B.M. was supported by grants OTKA 73430 and 103983. A.Saj. was supported by a Finnish Professorpool (Paulo Foundation) Grant. The Lithuanian sampling was supported by the LITGEN project (VP1-3.1-ŠMM-07-K-01-013), funded by the European Social Fund under the Global Grant Measure. A.S. was supported by Spanish grants SAF2011-26983 and EM 2012/ 045. O.U. was supported by Ukrainian SFFS grant F53.4/071. S.A.T. was supported by NIH Pioneer Award 8DP1ES022577-04 and NSF HOMINID award BCS-0827436. K.T. was supported by an Indian CSIR Network Project (GENESIS: BSC0121). L.S. was supported by an Indian CSIR Bhatnagar Fellowship. R.V., M.M., J.P. and E.M. were supported by the European Union Regional Development Fund through the Centre of Excellence in Genomics to the Estonian Biocentre and University of Tartu and by an Estonian Basic Research grant SF0270177As08. M.M. was additionally supported by Estonian Science Foundation grant number 8973. J.G.S. and M.S. were supported by NIH grant GM40282. P.H.S. and E.E.E. were supported by NIH grants HG004120 and 
HG002385. D.R. and N.P. were supported by NSF HOMINID award BCS-1032255 and $\mathrm{NIH}$ grant GM100233. D.R. and E.E.E. are Howard Hughes Medical Institute investigators. This project has been funded in part with federal funds from the National Cancer Institute, National Institutes of Health, under contract HHSN26120080001E. The content of this publication does not necessarily reflect the views or policies of the Department of Health and Human Services, nor does mention of trade names, commercial products, or organizations imply endorsement by the US Government This Research was supported in part by the Intramural Research Program of the NIH, National Cancer Institute, Center for Cancer Research.

Author Contributions B.B., E.E.E., J.Bu., M.S., S.P., J.Ke., D.R. and J.Kr. supervised the study. I.L., N.P., A.M., G.R., S.M., K.K., P.H.S., J.G.S., S.C., M.L., Q.F., H.L., C.dF., K.P., W.H., M.Met., M.Mey. and D.R. analysed genetic data. F.H., E.F., D.D., M.F., J.-M.G., J.W., A.C. and J.Kr. obtained human remains. A.M., C.E., R.Bo., K.I.B., S.S., C.P., N.R. and J.Kr. processed ancient DNA. I.L., N.P., S.N., N.R., G.A., H.A.B., G.Ba., E.B., O.B., R.Ba., G.Be., H.B.-A., J.Be. F.Be., C.M.B., F.Br., G.B.J.B., F.C., M.C., D.E.C.C., D.Cor., L.D., G.vD., S.D., J.-M.D., S.A.F., I.G.R., M.G., M.H., B.M.H., T.H., U.H., A.R.J., S.K.-Y., R.Kh., E.K., R.Ki., T.K., W.K., V.K., A.K., L.L., S.L., T.L., R.W.M., B.M., E.M., J.Mol., J.Mou., K.N., D.N., T.N., L.O., J.P., F.P., O.P., V.R., F.R., I.R., R.R., H.S., A.Saj., A.Sal., E.B.S., A.Tar., D.T., S.T., I.U., O.U., R.Va., M.Vi., M.Vo., C.A.W., L.Y., P.Z., T.Z., C.C., M.G.T., A.R.-L., S.A.T., L.S., K.T., R.Vi., D.Com., R.S., M.Met., S.P. and D.R. assembled the genotyping dataset. I.L., N.P., D.R. and J.Kr. wrote the manuscript with help from all co-authors.

Author Information The aligned sequences are available through the European Nucleotide Archive under accession number PRJEB6272. The fully public version of the Human Origins dataset can be found at (http://genetics.med.harvard.edu/ reichlab/Reich_Lab/Datasets.html). The full version of the dataset (including additional samples) is available to researchers who send a signed letter to D.R. indicating that they will abide by specified usage conditions (Supplementary Information section 9). Reprints and permissions information is available at www.nature.com/reprints. The authors declare competing financial interests: details are available in the online version of the paper. Readers are welcome to comment on the online version of the paper. Correspondence and requests for materials should be addressed to D.R. (reich@genetics.med.harvard.edu) or J.Kr. (johannes.krause@uni-tuebingen.de).

Iosif Lazaridis ${ }^{1,2}$, Nick Patterson ${ }^{2}$, Alissa Mittnik ${ }^{3}$, Gabriel Renaud ${ }^{4}$, Swapan Mallick ${ }^{1,2}$, Karola Kirsanow $^{5}$, Peter H. Sudmant ${ }^{6}$, Joshua G. Schraiber ${ }^{6,7}$, Sergi Castellano ${ }^{4}$, Mark Lipson ${ }^{8}$, Bonnie Berger ${ }^{2,8}$, Christos Economou, ${ }^{9}$, Ruth Bollongino ${ }^{5}$, Qiaomei Fu ${ }^{1,4,10}$ Kirsten I. Bos ${ }^{3}$, Susanne Nordenfelt ${ }^{1,2}$, Heng $\mathrm{Li}^{1,2}$, , Cesare de Filippo ${ }^{4}$, Kay Prüfer ${ }^{4}$, ' Susanna Sawyer ${ }^{4}$, Cosimo Posth ${ }^{3}$, Wolfgang Haak ${ }^{11}$, Fredrik Hallgren ${ }^{12}$,

Elin Fornander ${ }^{12}$, Nadin Rohland ${ }^{1,2}$, Dominique Delsate ${ }^{13,14}$, Michael Francken ${ }^{15}$, Jean-Michel Guinet ${ }^{13}$, Joachim Wahl ${ }^{16}$, George Ayodo ${ }^{17}$, Hamza A. Babiker ${ }^{18,19}$, Graciela Bailliet $^{20}$, Elena Balanovska ${ }^{21}$, Oleg Balanovsky ${ }^{21,22}$, Ramiro Barrantes ${ }^{23}$ Gabriel Bedoya ${ }^{24}$, Haim Ben-Ami ${ }^{25}$, Judit Ben ${ }^{26}$, Fouad Berrada ${ }^{27}$, Claudio M. Bravi $^{20}$, Francesca Brisighelli ${ }^{28}$, George B. J. Busby ${ }^{29,30}$, Francesco Cali ${ }^{31}$, Mikhail Churnosov $^{32}$, David E. C. Cole ${ }^{33}$, Daniel Corach ${ }^{34}$, Larissa Damba ${ }^{35}$, George van Driem $^{36}$, Stanislav Dryomov ${ }^{37}$, Jean-Michel Dugoujon ${ }^{38}$, Sardana A. Fedorova ${ }^{39}$, Irene Gallego Romero ${ }^{40}$, Marina Gubina ${ }^{35}$, Michael Hammer ${ }^{41}$, Brenna M. Henn ${ }^{42}$, Tor Hervig ${ }^{4}$, Ugur Hodoglugil ${ }^{44}$, Aashish R. Jha ${ }^{40}$, Sena Karachanak-Yankova ${ }^{45}$ Rita Khusainova ${ }^{46,47}$. Elza Khusnutdinova ${ }^{46,47}$, Rick Kittles ${ }^{48}$, Toomas Kivisild ${ }^{49}$ William Klitz ${ }^{7}$, Vaidutis Kučinskas ${ }^{50}$, Alena Kushniarevich ${ }^{51}$, Leila Laredj ${ }^{52}$, Sergey Litvinov $^{46,47,51}$, Theologos Loukidis ${ }^{53} \uparrow$, Robert W. Mahley ${ }^{54}$, Béla Melegh ${ }^{26}$, Ene ${\text { Metspalu }{ }^{55} \text {, Julio Molina }}^{56}$, Joanna Mountain ${ }^{57}$, Klemetti Näkkäläjärvi ${ }^{58}$, Desislava Nesheva $^{45}$, Thomas Nyambo ${ }^{59}$, Ludmila Osipova ${ }^{35}$, Jüri Parik ${ }^{55}$, Fedor Platonov ${ }^{60}$ Olga Posukh ${ }^{35}$, Valentino Romano ${ }^{61}$, Francisco Rothhammer $20,63,64$, Igor Rudan ${ }^{65}$ Ruslan Ruizbakiev ${ }^{66}$., Hovhannes Sahakyan ${ }^{51,67}$, Antti Sajantila ${ }^{68,69}$, Antonio Salas ${ }^{70}$ Elena B. Starikovskaya ${ }^{37}$, Ayele Tarekegn ${ }^{71}$, Draga Toncheva ${ }^{45}$, Shahlo Turdikulova ${ }^{72}$, Ingrida Uktveryte ${ }^{50}$, Olga Utevska ${ }^{73}$, René Vasquez ${ }^{74,75}$, Mercedes Villena ${ }^{74,75}$, Mikhail Voevoda ${ }^{35,76,77}$, Cheryl A. Winkler ${ }^{78}$, Levon Yepiskoposyan ${ }^{67}$, Pierre Zalloua $^{79,80}$, Tatijana Zemunik ${ }^{81}$, Alan Cooper ${ }^{11}$, Cristian Capelli ${ }^{29}$, Mark G. Thomas ${ }^{53}$ Andres Ruiz-Linares ${ }^{53}$, Sarah A. Tishkoff ${ }^{82}$, Lalji Singh ${ }^{33}+$, Kumarasamy Thangaraj ${ }^{83}$, Richard Villems ${ }^{51,55,84}$, David Comas ${ }^{85}$, Rem Sukernik ${ }^{37}$, Mait Metspalu ${ }^{51}$, Matthias Meyer ${ }^{4}$, Evan E. Eichler 6,86 , Joachim Burger ${ }^{5}$, Montgomery Slatkin ${ }^{7}$, Svante Pääbo ${ }^{4}$, Janet Kelso ${ }^{4}$, David Reich ${ }^{1,2,87}$ \& Johannes Krause ${ }^{3,88,89}$

${ }^{1}$ Department of Genetics, Harvard Medical School, Boston, Massachusetts 02115, USA 2Broad Institute of Harvard and MIT, Cambridge, Massachusetts 02142, USA. ${ }^{3}$ Institute for Archaeological Sciences, University of Tübingen, Tübingen 72074, Germany. ${ }^{4}$ Max Planck Institute for Evolutionary Anthropology, Leipzig 04103, Germany. Institute of Anthropology, Johannes Gutenberg University Mainz, Mainz D-55128, Germany. ${ }^{6}$ Department of Genome Sciences, University of Washington, Seattle, Washington 98195 , USA. ${ }^{7}$ Department of Integrative Biology, University of California, Berkeley, California 94720-3140, USA. ${ }^{8}$ Department of Mathematics and Computer Science and Artificial Intelligence Laboratory, Massachusetts Institute of Technology, Cambridge,

Massachusetts 02139, USA. ${ }^{9}$ Archaeological Research Laboratory, Stockholm University, 11418 , Sweden. ${ }^{10} \mathrm{Key}$ Laboratory of Vertebrate Evolution and Human Origins of Chinese Academy of Sciences, IVPP, CAS, Beijing 100049, China. ${ }^{11}$ Australian Centre for Ancient DNA and Environment Institute, School of Earth and Environmental Sciences, University of Adelaide, Adelaide, South Australia 5005. Australia. ${ }^{12}$ The Cultural Heritage

Foundation, Västerås 722 12, Sweden. ${ }^{13}$ National Museum of Natural History, L-2160, Luxembourg. ${ }^{14}$ National Center of Archaeological Research, National Museum of History and Art, L-2345, Luxembourg. ${ }^{15}$ Department of Paleoanthropology, Senckenberg Center for Human Evolution and Paleoenvironment, University of Tübingen, Tübingen D-72070, Germany. ${ }^{16}$ State Office for Cultural Heritage Management Baden-Württemberg,
Osteology, Konstanz D-78467, Germany. ${ }^{17}$ Center for Global Health and Child Development, Kisumu 40100, Kenya. ${ }^{18}$ Institutes of Evolution, Immunology and Infection Research, School of Biological Sciences, University of Edinburgh, Edinburgh EH9 3JT, UK. ${ }^{19}$ Biochemistry Department, Faculty of Medicine, Sultan Qaboos University, Alkhod, Muscat 123, Oman. ${ }^{20}$ Laboratorio de Genética Molecular Poblacional, Instituto Multidisciplinario de Biología Celular (IMBICE), CCT-CONICET \& CICPBA, La Plata, B1906APO, Argentina. ${ }^{21}$ Research Centre for Medical Genetics, Moscow 115478, Russia. ${ }^{22}$ Vavilov Institute for General Genetics, Moscow 119991, Russia. ${ }^{23}$ Escuela de Biología, Universidad de Costa Rica, San José 2060, Costa Rica. ${ }^{24}$ Institute of Biology, Research group GENMOL, Universidad de Antioquia, Medellín, Colombia. ${ }^{25}$ Rambam Health Care Campus, Haifa 31096, Israel. ${ }^{26}$ Department of Medical Genetics and Szentagothai Research Center, University of Pécs, Pécs H-7624, Hungary. ${ }^{27}$ Al Akhawayn University in Ifrane (AUI), School of Science and Engineering, Ifrane 53000, Morocco. ${ }^{28}$ Forensic Genetics Laboratory, Institute of Legal Medicine, Università Cattolica del Sacro Cuore, Rome 00168, Italy. ${ }^{29}$ Department of Zoology, University of Oxford, Oxford OX1 3PS, UK. ${ }^{30}$ Wellcome Trust Centre for Human Genetics, University of Oxford, Oxford OX3 7BN, UK. ${ }^{31}$ Laboratorio di Genetica Molecolare, IRCCS Associazione Oasi Maria SS, Troina 94018 Italy. ${ }^{32}$ Belgorod State University, Belgorod 308015, Russia. ${ }^{33}$ Department of Laboratory Medicine and Pathobiology, University of Toronto, Toronto, Ontario M5G 1L5, Canada. ${ }^{34}$ Servicio de Huellas Digitales Genéticas, School of Pharmacy and Biochemistry, Universidad de Buenos Aires, 1113 CABA, Argentina. ${ }^{35}$ Institute of Cytology and Genetics, Siberian Branch of Russian Academy of Sciences, Novosibirsk 630090, Russia. ${ }^{36}$ Institute of Linguistics, University of Bern, Bern $\mathrm{CH}-3012$, Switzerland. ${ }^{37}$ Laboratory of Human Molecular Genetics, Institute of Molecular and Cellular Biology, Russian Academy of Science, Siberian Branch, Novosibirsk 630090, Russia. ${ }^{38}$ Anthropologie Moléculaire et Imagerie de Synthèse, CNRS UMR 5288, Université Paul Sabatier Toulouse III, Toulouse 31000 , France. ${ }^{39}$ North-Eastern Federal University and Yakut Research Center of Complex Medical Problems, Yakutsk 677013, Russia. ${ }^{40}$ Department of Human Genetics, University of Chicago, Chicago, Illinois 60637, USA. ${ }^{41}$ ARL Division of Biotechnology, University of Arizona, Tucson, Arizona 85721, USA. ${ }^{42}$ Department of Ecology and Evolution, Stony Brook University, Stony Brook, New York 11794, USA. ${ }^{43}$ Department of Clinical Science, University of Bergen, Bergen 5021, Norway. ${ }^{44}$ NextBio, Illumina, Santa Clara, California 95050, USA. ${ }^{45}$ Department of Medical Genetics, National Human Genome Center, Medical University Sofia, Sofia 1431, Bulgaria. ${ }^{46}$ Institute of Biochemistry and Genetics, Ufa Research Centre, Russian Academy of Sciences, Ufa 450054, Russia. ${ }^{47}$ Department of Genetics and Fundamental Medicine, Bashkir State University, Ufa 450074, Russia. ${ }^{48}$ College of Medicine, University of Arizona, Tucson, Arizona 85724, USA. ${ }^{49}$ Division of Biological Anthropology, University of Cambridge, Cambridge CB2 $1 \mathrm{QH}$, UK. ${ }^{50}$ Department of Human and Medical Genetics, Vilnius University, Vilnius LT-08661, Lithuania. ${ }^{51}$ Estonian Biocentre, Evolutionary Biology group, Tartu, 51010, Estonia. ${ }^{52}$ Translational Medicine and Neurogenetics, Institut de Génétique et de Biologie Moléculaire et Cellulaire, Illkirch 67404, France. ${ }^{53}$ Department of Genetics, Evolution and Environment, University College London, London WC1E 6BT, UK. ${ }^{54}$ Gladstone Institutes, San Francisco, California 94158, USA. ${ }^{55}$ Department of Evolutionary Biology, University of Tartu, Tartu 51010, Estonia. ${ }^{56}$ Centro de Investigaciones Biomédicas de Guatemala, Ciudad de Guatemala, Guatemala. ${ }^{57}$ Research Department, 23andMe, Mountain View, California 94043, USA. ${ }^{58}$ Cultural Anthropology Program, University of Oulu, Oulu 90014, Finland. ${ }^{59}$ Department of Biochemistry, Muhimbili University of Health and Allied Sciences, Dar es Salaam 65001 Tanzania. ${ }^{60}$ Research Institute of Health, North-Eastern Federal University, Yakutsk 677000, Russia. ${ }^{61}$ Dipartimento di Fisica e Chimica, Università di Palermo, Palermo 90128, Italy. ${ }^{62}$ Instituto de Alta Investigación, Universidad de Tarapacá, Arica 1000000 Chile. ${ }^{6}$ Programa de Genética Humana ICBM Facultad de Medicina Universidad de Chile, Santiago 8320000 , Chile ${ }^{64}$ Centro de Investigaciones del Hombre en el Desierto, Arica 1000000 , Chile. ${ }^{6}$ Centre for Population Health Sciences, The University of Edinburgh Medical School, Edinburgh EH8 9AG, UK. ${ }^{66}$ Institute of Immunology, Academy of Science, Tashkent 70000, Uzbekistan. ${ }^{67}$ Laboratory of Ethnogenomics, Institute of Molecular Biology, National Academy of Sciences of Armenia, Yerevan 0014, Armenia.

${ }^{68}$ Department of Forensic Medicine, Hjelt Institute, University of Helsinki, Helsinki 00014 Finland. ${ }^{69}$ Institute of Applied Genetics, Department of Molecular and Medical Genetics, University of North Texas Health Science Center, Fort Worth, Texas 76107, USA.

${ }^{70}$ Unidade de Xenética, Departamento de Anatomía Patolóxica e Ciencias Forenses, and Instituto de Ciencias Forenses, Grupo de Medicina Xenómica (GMX), Facultade de Medicina, Universidade de Santiago de Compostela, Galcia 15872, Spain. ${ }^{71}$ Research Fellow, Henry Stewart Group, Russell House, London WC1A 2HN, UK. ${ }^{72}$ Institute of Bioorganic Chemistry Academy of Sciences Republic of Uzbekistan, Tashkent 100125, Uzbekistan. ${ }^{73}$ Department of Genetics and Cytology, V. N. Karazin Kharkiv National University, Kharkiv 61077, Ukraine. ${ }^{74}$ Instituto Boliviano de Biología de la Altura, Universidad Mayor de San Andrés, 5912 La Paz, Bolivia. ${ }^{75}$ Universidad Autonoma Tomás Frías, Potosí, Bolivia. ${ }^{76}$ Institute of Internal Medicine, Siberian Branch of Russian Academy of Medical Sciences, Novosibirsk 630089, Russia. ${ }^{77}$ Novosibirsk State University, Novosibirsk 630090 , Russia. ${ }^{78}$ Basic Research Laboratory, NCI, NIH, Frederick National Laboratory, Leidos Biomedical, Frederick, Maryland 21702. USA. ${ }^{79}$ Lebanese American University, School of Medicine, Beirut 13-5053, Lebanon. ${ }^{80}$ Harvard School of Public Health, Boston, Massachusetts 02115, USA. ${ }^{81}$ Department of Medical Biology, University of Split, School of Medicine, Split 21000, Croatia. ${ }^{82}$ Department of Biology and Genetics, University of Pennsylvania, Philadelphia, Pennsylvania 19104, USA. ${ }^{83}$ CSIR-Centre for Cellular and Molecular Biology, Hyderabad 500 007, India. ${ }^{84}$ Estonian Academy of Sciences, Tallinn 10130, Estonia. ${ }^{85}$ Institut de Biologia Evolutiva (CSIC-UPF), Departament de Ciències Experimentals i de la Salut, Universitat Pompeu Fabra Barcelona 08003, Spain. ${ }^{86}$ Howard Hughes Medical Institute, University of Washington, Seattle, Washington 98195, USA. ${ }^{87}$ Howard Hughes Medical Institute, Harvard Medical School, Boston, Massachusetts 02115, USA. ${ }^{88}$ Senckenberg Centre for Human Evolution and Palaeoenvironment, University of Tübingen, 72070 Tübingen, Germany. ${ }^{89}$ Max Planck Institut für Geschichte und Naturwissenschaften, Jena 07745, Germany. †Present addresses: Amgen, 33 Kazantzaki Str, llioupolis 16342, Athens, Greece (T.L.); Banaras Hindu University, Varanasi 221 005, India (L.S.). \$Deceased. 


\section{METHODS}

Archaeological context, sampling and DNA extraction. The Loschbour sample stems from a male skeleton excavated in 1935 at the Loschbour rock shelter in Heffingen, Luxembourg. The skeleton was AMS radiocarbon dated to 7,205 \pm 50 years before present (OxA-7738; 6,220-5,990 cal. BC) ${ }^{31}$. At the Palaeogenetics Laboratory in Mainz, material for DNA extraction was sampled from tooth 16 (an upper right M1 molar) after irradiation with ultraviolet light, surface removal, and pulverization in a mixer mill. DNA extraction took place in the palaeogenetics facilities in the Institute for Archaeological Sciences at the University of Tübingen. Three extracts were made in total, one from $80 \mathrm{mg}$ of powder using an established silica based protocol ${ }^{32}$ and two additional extracts from $90 \mathrm{mg}$ of powder each with a protocol optimized for the recovery of short DNA molecules ${ }^{33}$.

The Stuttgart sample was taken from a female skeleton excavated in 1982 at the site Viesenhäuser Hof, Stuttgart-Mühlhausen, Germany. It was attributed to the Linearbandkeramik $(5,500-4,800 \mathrm{BC})$ through associated pottery artefacts and the chronology was corroborated by radiocarbon dating of the stratigraphy ${ }^{34}$. Both sampling and DNA extraction took place in the Institute for Archaeological Sciences at the University of Tübingen. Tooth 47 (a lower right M2 molar) was removed and material from the inner part was sampled with a sterile dentistry drill. An extract was made using $40 \mathrm{mg}$ of bone powder ${ }^{33}$.

The Motala individuals were recovered from the site of Kanaljorden in the town of Motala, Östergötland, Sweden, excavated between 2009 and 2013. The human remains at this site are represented by several adult skulls and one infant skeleton. All individuals are part of a ritual deposition at the bottom of a small lake. Direct radiocarbon dates on the remains range between 7,013 \pm 76 and 6,701 \pm 64 вP $(6,361-$ 5,516 cal. BC), corresponding to the late Middle Mesolithic of Scandinavia. Samples were taken from the teeth of the nine best preserved skulls, as well as a femur and tibia. Bone powder was removed from the inner parts of the teeth or bones with a sterile dentistry drill. DNA from $100 \mathrm{mg}$ of bone powder was extracted ${ }^{35}$ in the ancient DNA laboratory of the Archaeological Research Laboratory, Stockholm.

Library preparation. Illumina sequencing libraries were prepared using either doubleor single-stranded library preparation protocols ${ }^{36,37}$ (Supplementary Information section 1). For high-coverage shotgun sequencing libraries, a DNA repair step with uracil DNA glycosylase (UDG) and endonuclease VIII (endo VIII) treatment was included in order to remove uracil residues ${ }^{38}$. Size fractionation on a PAGE gel was also performed in order to remove longer DNA molecules that are more likely to be contaminants ${ }^{37}$. Positive and blank controls were carried along during every step of library preparation.

Shotgun sequencing and read processing. All non-UDG-treated libraries were sequenced either on an Illumina Genome Analyzer IIx with $2 \times 76+7$ cycles for the Loschbour and Motala libraries, or on an Illumina MiSeq with $2 \times 150+8+8$ cycles for the Stuttgart library. We followed the manufacturer's protocol for multiplex sequencing. Raw overlapping forward and reverse reads were merged and filtered for quality ${ }^{39}$ and mapped to the human reference genome (hg19/GRCh37/ 1000 Genomes) using the Burrows-Wheeler Aligner (BWA ${ }^{40}$ (Supplementary Information section 2). For deeper sequencing, UDG-treated libraries of Loschbour were sequenced on 3 Illumina HiSeq 2000 lanes with 50-bp single-end reads, 8 Illumina HiSeq 2000 lanes of 100-bp paired-end reads and 8 Illumina HiSeq 2500 lanes of 101-bp paired-end reads. The UDG-treated library for Stuttgart was sequenced on 8 HiSeq 2000 lanes of 101-bp paired-end reads. The UDG-treated libraries for Motala were sequenced on 8 HiSeq 2000 lanes of 100-bp paired-end reads, with 4 lanes each for two pools (one of 3 individuals and one of 4 individuals). We also sequenced an additional 8 HiSeq 2000 lanes for Motala12, the Motala sample with the highest percentage of endogenous human DNA. For the Loschbour and Stuttgart high coverage individuals, diploid genotype calls were obtained using the Genome Analysis Toolkit (GATK) ${ }^{41}$

Enrichment of mitochondrial DNA and sequencing. To test for DNA preservation and mtDNA contamination, non-UDG-treated libraries of Loschbour and all Motala samples were enriched for human mitochondrial DNA using a bead-based capture approach with present-day human DNA as bait ${ }^{42}$. UDG-treatment was omitted in order to allow characterization of damage patterns typical for ancient $\mathrm{DNA}^{10}$ The captured libraries were sequenced on an Illumina Genome Analyzer IIx platform with $2 \times 76+7$ cycles and the resulting reads were merged and quality filtered ${ }^{39}$. The sequences were mapped to the Reconstructed Sapiens Reference Sequence, RSRS ${ }^{43}$, using a custom iterative mapping assembler, $\mathrm{MIA}^{44}$ (Supplementary Information section 4).

Contamination estimates. We assessed if the sequences had the characteristics of authentic ancient DNA using four approaches. First, we searched for evidence of contamination by determining whether the sequences mapping to the mitochondrial genome were consistent with deriving from more than one individual ${ }^{44,45}$. Second, for the high-coverage Loschbour and Stuttgart genomes, we used a maximum-likelihoodbased estimate of autosomal contamination that uses variation at sites that are fixed in the 1000 Genomes data to estimate error, heterozygosity and contamination ${ }^{46}$ simultaneously. Third, we estimated contamination based on the rate of polymorphic sites on the $\mathrm{X}$ chromosome of the male Loschbour individual ${ }^{47}$ (Supplementary Information section 3) Fourth, we analysed non-UDG treated reads mapping to the RSRS to search for ancient DNA-typical damage patterns resulting in $\mathrm{C} \rightarrow \mathrm{T}$ changes at the $5^{\prime}$-end of the molecule ${ }^{10}$ (Supplementary Information section 3 ). Phylogenetic analysis of the mitochondrial genomes. All nine complete mitochondrial genomes that fulfilled the criteria of authenticity were assigned to haplogroups using Haplofind ${ }^{48}$. A Maximum Parsimony tree including present-day humans and previously published ancient mtDNA sequences was generated with $\mathrm{MEGA}^{49}$. The effect of branch shortening due to a lower number of substitutions in ancient lineages was studied by calculating the nucleotide edit distance to the root for all haplogroup R sequences (Supplementary Information section 4).

Sex determination and $Y$-chromosome analysis. We assessed the sex of all sequenced individuals by using the ratio of (chrY) to (chrY $+\mathrm{chrX})$ aligned reads ${ }^{50}$. We downloaded a list of Y-chromosome SNPs curated by the International Society of Genetic Genealogy (ISOGG, http://www.isogg.org) v. 9.22 (accessed Feb. 18, 2014) and determined the state of the ancient individuals at positions where a single allele was observed and MAPQ $\geq 30$. We excluded $\mathrm{C} / \mathrm{G}$ or $\mathrm{A} / \mathrm{T} \mathrm{SNPs}$ due to uncertainty about the polarity of the mutation in the database. The ancient individuals were assigned haplogroups based on their derived state (Supplementary Information section 5). We also used BEAST v1.7.51 (ref. 51) to assess the phylogenetic position of Loschbour using 623 males from around the world with 2,799 variant sites across $500 \mathrm{~kb}$ of non-recombining Y-chromosome sequence ${ }^{52}$ (Supplementary Information section 5).

Estimation of Neanderthal admixture. We estimate Neanderthal admixture in ancient individuals with the $f_{4}$ ratio or $S$ statistic ${ }^{8,53,54} \hat{\alpha}=f_{4}$ (Altai, Denisova; Test Yoruba) $/ f_{4}$ (Altai, Denisova; Vindija, Yoruba) which uses whole genome data from Altai, a high coverage $(52 \times)$ Neanderthal genome sequence ${ }^{55}$, Denisova, a high coverage sequence ${ }^{37}$ from another archaic human population $(31 \times)$, and Vindija, a low coverage $(1.3 \times)$ Neanderthal genome from a mixture of three Neanderthal individuals from Vindija Cave in Croatia ${ }^{53}$

Inference of demographic history and inbreeding. We used the Pairwise Sequen tially Markovian Coalescent (PSMC) ${ }^{56}$ to infer the size of the ancestral population of Stuttgart and Loschbour as it changed over time. This analysis requires high quality diploid genotype calls and cannot be performed in the low-coverage Motala samples. To determine whether the low effective population size inferred for Loschbour is due to recent inbreeding, we plotted the time-to-most-recent common ancestor (TMRCA) along each of chromosomes 1-22 to detect runs of low TMRCA.

Analysis of segmental duplications and copy number variants. We built readdepth based copy number maps for the Loschbour, Stuttgart and Motala12 genomes in addition to the Denisova and Altai Neanderthal genome and 25 deeply sequenced modern genomes ${ }^{55}$ (Supplementary Information section 7). We built these maps by aligning reads, subdivided into their non-overlapping 36-bp constituents, against the reference genome using the mrsFAST aligner ${ }^{57}$, and renormalizing read-depth for local GC content. We estimated copy numbers in windows of 500 unmasked base pairs slid at 100-bp intervals across the genome. We called copy number variants using a scale space filter algorithm. We genotyped variants of interest and compared the genotypes to those from individuals sequenced as part of the 1000 Genomes Project ${ }^{58}$.

Phenotypic inference. We inferred likely phenotypes (Supplementary Information section 8) by analysing DNA polymorphism data in the VCF format ${ }^{59}$ using VCFtools (http://vcftools.sourceforge.net). For the Loschbour and Stuttgart individuals, we included data from sites not flagged as LowQuality, with genotype quality (GQ) of $\geq 30$, and SNP quality (QUAL) of $\geq 50$. For Motala12, which is of lower coverage, we included sites having at least $2 \times$ coverage and that passed visual inspection of the local alignment using samtools tview (http://samtools.sourceforge.net) ${ }^{60}$.

Human Origins data set. We report new data on 1,615 present-day humans from 147 worldwide populations genotyped on the Affymetrix Human Origins array, all of whom provided informed consent consistent with studies of population history. The Human Origins array consists of 14 panels of SNPs for which the ascertainment is well known ${ }^{8,61}$. All population genetics analysis were carried out on a set of 594,924 autosomal SNPs, after restricting to sites that had $>90 \%$ completeness across 7 different batches of sequencing, and that had $>97.5 \%$ concordance with at least one of two subsets of samples for which whole-genome sequencing data were also available. We generated our full data set by merging the newly collected data with previously reported data, resulting in 2,722 individuals (208 populations), which we filtered to 2,345 individuals (203 populations) after removing outlier individuals or relatives based on visual inspection of PCA plots ${ }^{14,62}$ or modelbased clustering analysis ${ }^{13}$. Whole genome amplified (WGA) individuals were not used in analysis, except for a Saami individual who we included because of the special interest of this population for northeastern European population history (Extended Data Fig. 7). 
ADMIXTURE analysis. We merged all Human Origins genotype data with whole genome sequencing data from Loschbour, Stuttgart, MA1, Motala12, Motala_merge, and LaBraña. We then thinned the resulting data set to remove SNPs in linkagedisequilibrium with PLINK 1.07 (ref. 63), using a window size of 200 SNPs advanced by 25 SNPs and an $r^{2}$ threshold of 0.4. We ran ADMIXTURE 1.23 (refs 13, 64) for 100 replicates with different starting random seeds, default fivefold cross-validation, and varying the number of ancestral populations $K$ between 2 and 20. We assessed clustering quality using CLUMPP ${ }^{65}$. We used the ADMIXTURE results to identify a set of 59 'west Eurasian' (European/Near Eastern) populations based on values of a west Eurasian ancestral population at $K=3$ (Supplementary Information section 9). We also identified 15 populations for use as 'non-west Eurasian outgroups' based on their having at least 10 individuals and no evidence of European or Near Eastern admixture at $K=11$, the lowest $K$ for which Near Eastern/European-maximized ancestral populations appeared consistently across all 100 replicates.

Principal components analysis. We used smartpca ${ }^{14}$ (version: 10210) from EIGENSOFT $^{62,66}$ 5.0.1 to carry out principal components analysis (PCA) (Supplementary Information section 10). We performed PCA on a subset on individuals and then projected others using the lsqproject: YES option that gives an unbiased inference of the position of samples even in the presence of missing data

(especially important for ancient DNA).
$\boldsymbol{f}_{3}$ statistics. We use the $f_{3}$ statistic $^{8} f_{3}\left(\right.$ Test $;$ Ref $\left._{1}, \operatorname{Ref}_{2}\right)=\frac{1}{N} \sum_{i=1}^{N}\left(t_{i}-r_{1, i}\right)\left(t_{i}-r_{2, i}\right)$, where $t_{i}, r_{1, \mathrm{i}}$ and $r_{2, \mathrm{i}}$ are the allele frequencies for the $i^{\text {th }}$ SNP in populations Test, $R e f_{1}, R e f_{2}$, respectively, to determine if there is evidence that the Test population is derived from admixture of populations related to $R e f_{1}$ and $R e f_{2}$ (Supplementary Information section 11). A significantly negative statistic provides unambiguous evidence of mixture in the Test population ${ }^{8}$. We allow $R e f_{1}$ and $R e f_{2}$ to be any Human Origins population with 4 or more individuals, or Loschbour, Stuttgart, MA1, Motala12, LaBraña. We assess significance of the $f_{3}$ statistics using a block jackknife ${ }^{67}$ and a block size of $5 \mathrm{cM}$. We report significance as the number of standard errors by which the statistic differs from zero ( $Z$-score). We also perform an analysis in which we constrain the reference populations to be (1) EEF (Stuttgart) and WHG (Loschbour or LaBraña), (2) EEF and a Near Eastern population, (3) EEF and ANE (MA1), or (4) any two present-day populations, and compute a $Z_{\mathrm{diff}}$ score between the lowest $f_{3}$ statistic observed in the data set, and the $f_{3}$ statistic observed for the specified pair. $\boldsymbol{f}_{4}$ statistics. We analyse $f_{4}$ statistics $^{8}$ of the form $f_{4}(A, B ; C, D)=\frac{1}{N} \sum_{i=1}^{N}\left(a_{i}-b_{i}\right)$ $\left(c_{i}-d_{i}\right)$ to assess if populations $\mathrm{A}, \mathrm{B}$ are consistent with forming a clade in an unrooted tree with respect to $\mathrm{C}, \mathrm{D}$. If they form a clade, the allele frequency differences between the two pairs should be uncorrelated and the statistic has an expected value of 0 . We set the outgroup $D$ to be a sub-Saharan African population or chimpanzee. We systematically tried all possible combinations of the ancient samples or 15 'non-west Eurasian outgroups' identified by ADMIXTURE analysis as A, B, C to determine their genetic affinities (Supplementary Information section 14). Setting A as a present-day test population and B as either Stuttgart or BedouinB, we documented relatedness to $\mathrm{C}=($ Loschbour or MA1) or $\mathrm{C}=(\mathrm{MA1}$ and Karitiana) or $\mathrm{C}=(\mathrm{MA} 1$ or Han $)$ (Extended Data Figs 4,5 and 7$)$. Setting $\mathrm{C}$ as a test population and (A, B) a pair from (Loschbour, Stuttgart, MA1) we documented differential relatedness to ancient populations (Extended Data Fig. 6). We computed $D$-statistics ${ }^{53}$ using transversion polymorphisms in whole genome sequence data ${ }^{55}$ to confirm robustness to ascertainment and ancient DNA damage (Extended Data Table 2). Minimum number of source populations for Europeans. We used $q \mathrm{pWave} \mathrm{e}^{16,17}$ to study the minimum number of source populations for a designated set of Europeans (Supplementary Information section 12). We use $f_{4}$ statistics of the form $X(l$, $r)=f_{4}\left(l_{0}, l ; r_{0}, r\right)$ where $l_{0}, r_{0}$ are arbitrarily chosen 'base' populations, and $l, r$ are other populations from two sets $L$ and $R$ respectively. If $X(l, r)$ has rank $r$ and there were $n$ waves of immigration into $R$ with no back-migration from $R$ to $L$, then $r+1 \leq n$. We set $L$ to include Stuttgart, Loschbour, MA1, Onge, Karitiana, Mbuti and $R$ to include 23 modern European populations who fit the model of Supplementary Information section 14 and had admixture proportions within the interval $[0,1]$ for the method with minimal modelling assumptions (Supplementary Information section 17).

Admixture proportions for Stuttgart in the absence of a Near Eastern ancient genome. We used Loschbour and BedouinB as surrogates for 'unknown huntergatherer' and Near Eastern (NE) farmer populations that contributed to Stuttgart (Supplementary Information section 13). Ancient Near Eastern ancestry in Stuttgart is estimated by the $f_{4}$ ratio ${ }^{8,15} f_{4}$ (Outgroup, $X$; Loschbour, Stuttgart) $/ f_{4}$ (Outgroup, $X ;$ Loschbour, NE). A complication is that BedouinB is a mixture of NE and African ancestry. We therefore subtracted ${ }^{17}$ the effects of African ancestry using estimates of the BedouinB African admixture proportion from ADMIXTURE (Supplementary Information section 9) or ALDER ${ }^{68}$.

Admixture graph modelling. We used ADMIXTUREGRAPH ${ }^{8}$ (version 3110) to model population relationships between Loschbour, Stuttgart, Onge, and Karitiana using Mbuti as an African outgroup. We assessed model fit using a block jackknife of differences between estimated and fitted $f$ statistics for the set of included populations (we expressed the fit as a $Z$ score). We determined that a model failed if $|Z|>3$ for at least one $f$ statistic. A basic tree model failed and we manually amended the model to test all possible models with a single admixture event, which also failed. Further manual amendment to include 2 admixture events resulted in 8 successful models, only one of which could be amended to also fit MA1 as an additional constraint. We successfully fit both the Iceman and LaBraña into this model as simple clades and Motala12 as a two-way mixture. We also fit present-day west Eurasians as clades, two-way mixtures, or three-way mixtures in this basic model, achieving a successful fit for a larger number of European populations $(n=26)$ as three-way mixtures. We estimated the individual admixture proportions from the fitted model parameters. To test if fitted parameters for different populations are consisten with each other, we jointly fit all pairs of populations $A$ and $B$ by modifying ADMIXTUREGRAPH to add a large constant $(10,000)$ to the variance term $f_{3}\left(A_{0}, A, B\right)$. By doing this, we can safely ignore recent gene flow within Europe that affects statistics that include both $A$ and $B$.

Ancestry estimates from $\boldsymbol{f}_{4}$-ratios. We estimate EEF ancestry using the $f_{4}$ ratio rit. $^{8,15}$ $f_{4}$ (Mbuti, Onge; Loschbour, European) $/ f_{4}$ (Mbuti, Onge; Loschbour, Stuttgart), which produces consistent results with ADMIXTUREGRAPH (Supplementary Information section 14). We use $f_{4}$ (Stuttgart, Loschbour; Onge MA1) $/ f_{4}$ (Mbuti, MA1; Onge, Loschbour) to estimate Basal Eurasian admixture into Stuttgart. We use $f_{4}$ (Stuttgart, Loschbour; Onge Karitiana) $/ \mathrm{f}_{4}$ (Stuttgart, Loschbour; Onge MA1) to estimate ANE mixture in Karitiana (Fig. 4). We use $f_{4}$ (Test, Stuttgart; Karitiana, Onge) $/ \mathrm{f}_{4}(\mathrm{MA1}$, Stuttgart; Karitiana, Onge) to lower bound ANE mixture into north Caucasian populations MixMapper analysis. We carried out MixMapper 2.0 (ref. 7) analysis, a semi-supervised admixture graph fitting technique. First, we infer a scaffold tree of population without strong evidence of mixture relative to each other (Mbuti, Onge, Loschbour and MA1). We do not include European populations in the scaffold as all had significantly negative $f_{3}$ statistics indicating admixture. We then ran MixMapper to infer the relatedness of the other ancient and present-day samples, fitting them onto the scaffold as two- or three-way mixtures. The uncertainty in all parameter estimates is measured by block bootstrap resampling of the SNP set (100 replicates with 50 blocks) TreeMix analysis. We applied TreeMix ${ }^{21}$ to Loschbour, Stuttgart, Motala12, and MA1 (ref. 3), LaBraña ${ }^{2}$ and the Iceman ${ }^{1}$, along with the present-day samples of Karitiana, Onge and Mbuti. We restricted the analysis to 265,521 Human Origins array sites after excluding any SNPs where there were no-calls in any of the studied individuals. The tree was rooted with Mbuti and standard errors were estimated using blocks of 500 SNPs. We repeated the analysis on whole-genome sequence data, rooting with chimp and replacing Onge with Dai as we did not have Onge whole genome sequence data ${ }^{55}$. We varied the number of migration events $(m)$ between 0 and 5 .

Inferring admixture proportions with minimal modelling assumptions. We devised a method to infer ancestry proportions from three ancestral populations (EEF, WHG, and ANE) without strong phylogenetic assumptions (Supplementary Information section 17). We rely on 15 'non-west Eurasian' outgroups and study $f_{4}$ (European, Stuttgart; $O_{1}, O_{2}$ ) which is expected to equal $\alpha \beta f_{4}$ (Loschbour, Stuttgart; $\left.O_{1}, O_{2}\right)+\alpha(1-\beta) f_{4}\left(\mathrm{MA} 1\right.$, Stuttgart; $\left.O_{1}, O_{2}\right)$ if European has $1-a$ ancestry from EEF and $\beta, 1-\beta$ ancestry from WHG and ANE respectively. This defines a system of $\left(\begin{array}{c}15 \\ 2\end{array}\right)=105$ equations with unknowns $\alpha \beta, \alpha(1-\beta)$, which we solve with least squares implemented in the function $l s f i t$ in $R$ to obtain estimates of $\alpha$ and $\beta$. We repeated this computation 22 times dropping one chromosome at a time ${ }^{20}$ to obtain block jackknife ${ }^{67}$ estimates of the ancestry proportions and standard errors, with block size equal to the number of SNPs per chromosome. We assessed consistency of the inferred admixture proportions with those derived from the ADMIXTUREGRAPH mode based on the number of standard errors between the two (Extended Data Table 1). Haplotype-based analyses. We used RefinedIBD from BEAGLE $4^{27}$ with the settings $i b d t r i m=20$ and $i b d w i n d o w=25$ to identify identity-by-descent (IBD) tracts: genomic segments or recently shared ancestry between Loschbour and Stuttgart and populations from the POPRES data set ${ }^{69}$. We kept all IBD tracts spanning at least 0.5 centimorgans $(\mathrm{cM})$ and with a LOD score $>3$ (Supplementary Information section 18). We also used ChromoPainter ${ }^{29}$ to study haplotype sharing between Loschbour and Stuttgart and present-day West Eurasian populations (SI19). We identified 495,357 SNPs that were complete in all individuals and phased the data using Beagle 4 (ref. 27) with parameters phase-its $=50$ and impute-its $=10$. We did not keep sites with missing data to avoid imputing modern alleles into the ancient individuals. We used both unlinked (-k 1000) and linked modes (estimating -n and -M by sampling $10 \%$ of individuals). We combined ChromoPainter output for chromosomes 1-22 using ChromoCombine ${ }^{29}$. We carried out a PCA of the co-ancestry matrix using fineSTRUCTURE ${ }^{29}$.

31. Delsate, D., Guinet, J.-M. \& Saverwyns, S. De l'ocre sur le crâne mésolithique (haplogroupe U5a) de Reuland-Loschbour (Grand-Duché de Luxembourg)? Bull. Soc. Préhist. Luxembourgeoise 31, 7-30 (2009). 
32. Rohland, N. \& Hofreiter, M. Ancient DNA extraction from bones and teeth. Nature Protocols 2, 1756-1762 (2007).

33. Dabney, J. et al. Complete mitochondrial genome sequence of a Middle Pleistocene cave bear reconstructed from ultrashort DNA fragments. Proc. Natl Acad. Sci. USA 110, 15758-15763 (2013).

34. Stäuble, H. Häuser und absolute Datierung der Ältesten Bandkeramik (Habelt, 2005).

35. Yang, D. Y., Eng, B., Waye, J. S., Dudar, J. C. \& Saunders, S. R. Improved DNA extraction from ancient bones using silica-based spin columns. Am. J. Phys. Anthropol. 105, 539-543 (1998).

36. Meyer, M. \& Kircher, M. Illumina sequencing library preparation for highly multiplexed target capture and sequencing. Cold Spring Harb. Protoc. http:// dx.doi.org/10.1101/prot5448 (2010).

37. Meyer, M. et al. A high-coverage genome sequence from an Archaic Denisovan individual. Science 338, 222-226 (2012).

38. Briggs, A. W. et al. Removal of deaminated cytosines and detection of in vivo methylation in ancient DNA. Nucleic Acids Res. 38, e87 (2010).

39. Kircher, M. Analysis of high-throughput ancient DNA sequencing data. Methods Mol. Biol. 480, 197-228 (2012).

40. Li, H. \& Durbin, R. Fast and accurate short read alignment with Burrows-Wheeler transform. Bioinformatics 25, 1754-1760 (2009).

41. McKenna, A. et al. The Genome Analysis Toolkit: a MapReduce framework for analyzing next-generation DNA sequencing data. Genome Res. 20, 1297-1303 (2010).

42. Maricic, T., Whitten, M. \& Pääbo, S. Multiplexed DNA sequence capture of mitochondrial genomes using PCR products. PLOS ONE 5, e14004 (2010).

43. Behar, D. M. et al. A Copernican reassessment of the human mitochondrial DNA tree from its root. Am. J. Hum. Genet. 90, 675-684 (2012).

44. Green, R. E. et al. A complete Neandertal mitochondrial genome sequence determined by high-throughput sequencing. Cell 134, 416-426 (2008).

45. $\mathrm{Fu}, \mathrm{Q}$. et al. A revised timescale for human evolution based on ancient mitochondrial genomes. Curr. Biol. 23, 553-559 (2013).

46. Fu, Q. et al. The complete genome sequence of a 45,000-year-old modern human from western Siberia. Nature (in the press).

47. Rasmussen, M. et al. An Aboriginal Australian genome reveals separate human dispersals into Asia. Science 334, 94-98 (2011).

48. Vianello, D. et al. HAPLOFIND: a new method for high-throughput mtDNA haplogroup assignment. Hum. Mutat. 34, 1189-1194 (2013).

49. Tamura, K. et al. MEGA5: molecular evolutionary genetics analysis using maximum likelihood, evolutionary distance, and maximum parsimony methods. Mol. Biol. Evol. 28, 2731-2739 (2011).

50. Skoglund, P., Storå, J., Götherström, A. \& Jakobsson, M. Accurate sex identification of ancient human remains using DNA shotgun sequencing. J. Archaeol. Sci. 40, 4477-4482 (2013)
51. Drummond, A. J. \& Rambaut, A. BEAST: Bayesian evolutionary analysis by sampling trees. BMC Evol. Biol. 7, 214 (2007).

52. Lippold, S. et al. Human paternal and maternal demographic histories: insights from high-resolution $Y$ chromosome and mtDNA sequences. Preprint at bioRxiv, http://dx.doi.org/10.1101/001792 (2014).

53. Green, R. E. et al. A draft sequence of the Neandertal genome. Science $\mathbf{3 2 8}$, 710-722 (2010)

54. Reich, D. et al. Genetic history of an archaic hominin group from Denisova Cave in Siberia. Nature 468, 1053-1060 (2010)

55. Prüfer, K. et al. The complete genome sequence of a Neanderthal from the Altai Mountains. Nature 505, 43-49 (2014).

56. Li, H. \& Durbin, R. Inference of human population history from individual whole-genome sequences. Nature 475, 493-496 (2011).

57. Hach, F. et al. mrsFAST: a cache-oblivious algorithm for short-read mapping. Nature Methods 7, 576-577 (2010).

58. The 1000 Genomes Project Consortium An integrated map of genetic variation from 1,092 human genomes. Nature 491, 56-65 (2012).

59. Danecek, P. et al. The variant call format and VCFtools. Bioinformatics 27, 2156-2158 (2011)

60. Li, H. The sequence alignment/map (SAM) format and SAMtools. Bioinformatics 25, 2078-2079 (2009)

61. Keinan, A., Mullikin, J. C., Patterson, N. \& Reich, D. Measurement of the human allele frequency spectrum demonstrates greater genetic drift in East Asians than in Europeans. Nature Genet. 39, 1251-1255 (2007).

62. Price, A. L. et al. Principal components analysis corrects for stratification in genome-wide association studies. Nature Genet. 38, 904-909 (2006).

63. Purcell, S. et al. PLINK: a tool set for whole-genome association and population-based linkage analyses. Am. J. Hum. Genet. 81, 559-575 (2007)

64. Alexander, D. H. \& Lange, K. Enhancements to the ADMIXTURE algorithm for individual ancestry estimation. BMC Bioinformatics 12, 246 (2011)

65. Jakobsson, M. \& Rosenberg, N. A. CLUMPP: a cluster matching and permutation program for dealing with label switching and multimodality in analysis of population structure. Bioinformatics 23, 1801-1806 (2007).

66. Price, A. L., Zaitlen, N. A., Reich, D. \& Patterson, N. New approaches to population stratification in genome-wide association studies. Nature Rev. Genet. 11, 459-463 (2010)

67. Busing, F. T. A., Meijer, E. \& Leeden, R. Delete-m Jackknife for Unequal m. Stat. Comput. 9, 3-8 (1999).

68. Loh, P.-R. et al. Inferring admixture histories of human populations using linkage disequilibrium. Genetics 193, 1233-1254 (2013).

69. Nelson, M. R. et al. The population reference sample, POPRES: a resource for population, disease, and pharmacological genetics research. Am. J. Hum. Genet. 83, 347-358 (2008). 


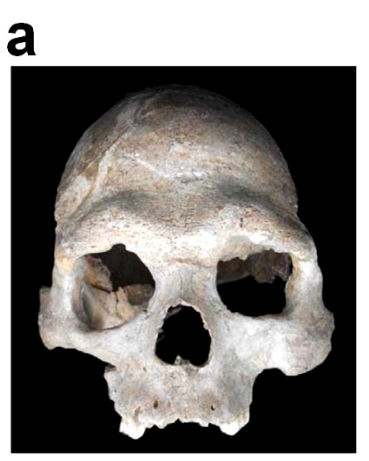

C

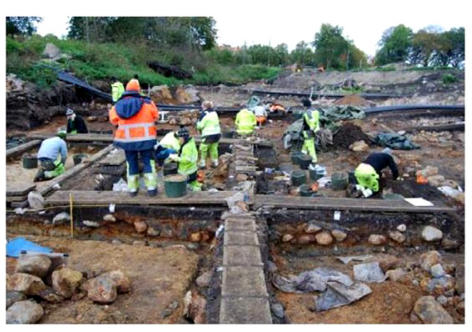

b

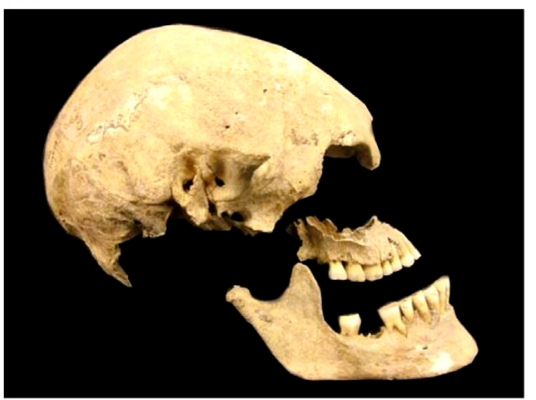

d

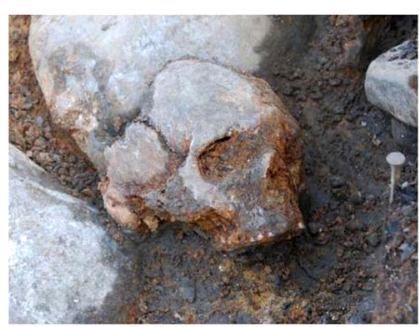

Extended Data Figure $1 \mid$ Photographs of analysed ancient samples. a, Loschbour skull. b, Stuttgart skull, missing the lower right M2 we sampled. c, Excavation at Kanaljorden in Motala, Sweden. d, Motala 1 in situ. 
a

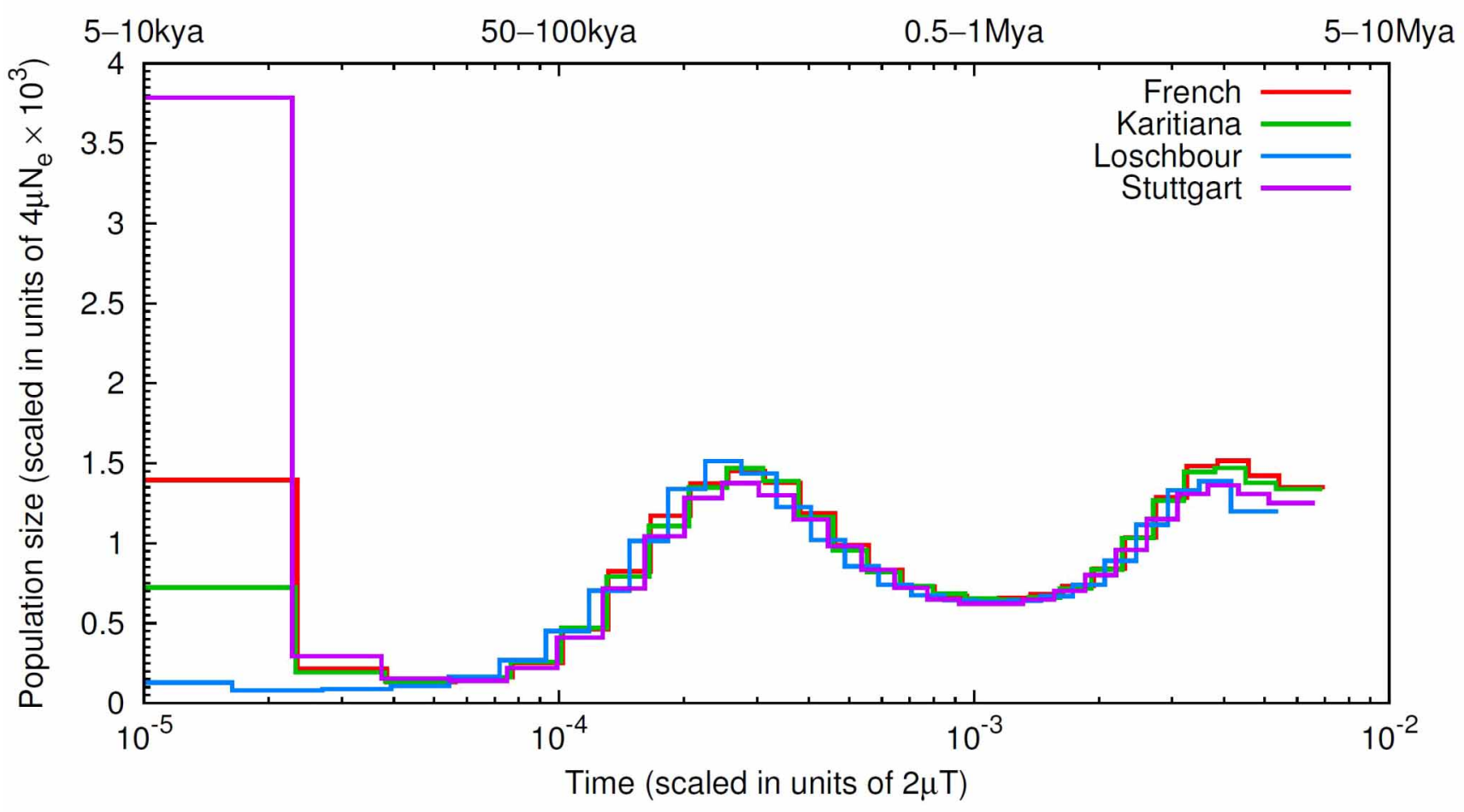

b

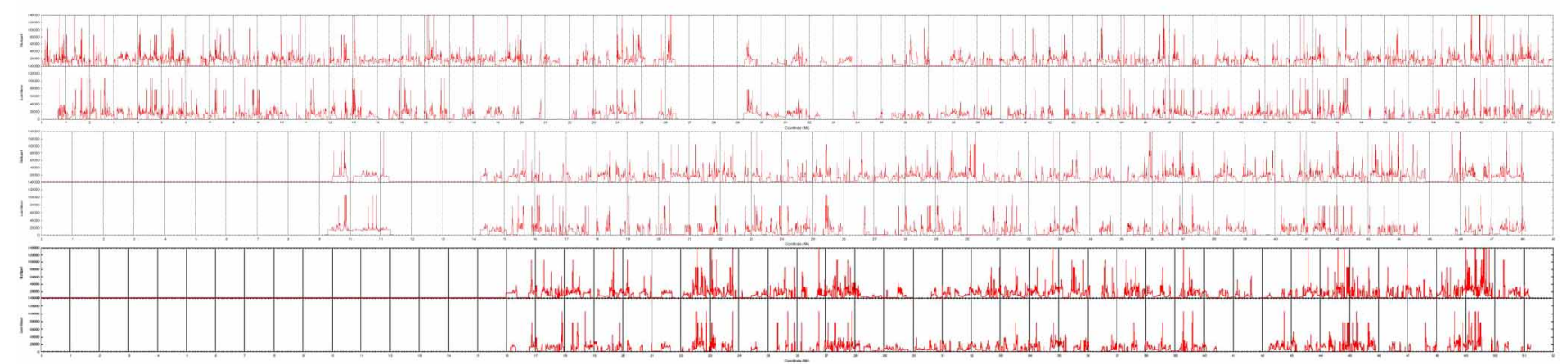

Extended Data Figure $2 \mid$ Pairwise sequential Markovian coalescent (PSMC) analysis. a, Inference of population size as a function of time, showing a very small recent population size over the most recent period in the ancestry of Loschbour (at least the last 5-10 thousand years). b. Inferred time since the most recent common ancestor from the PSMC for chromosomes 20, 21, 22 (top to bottom); Stuttgart is plotted on top and Loschbour at bottom. 


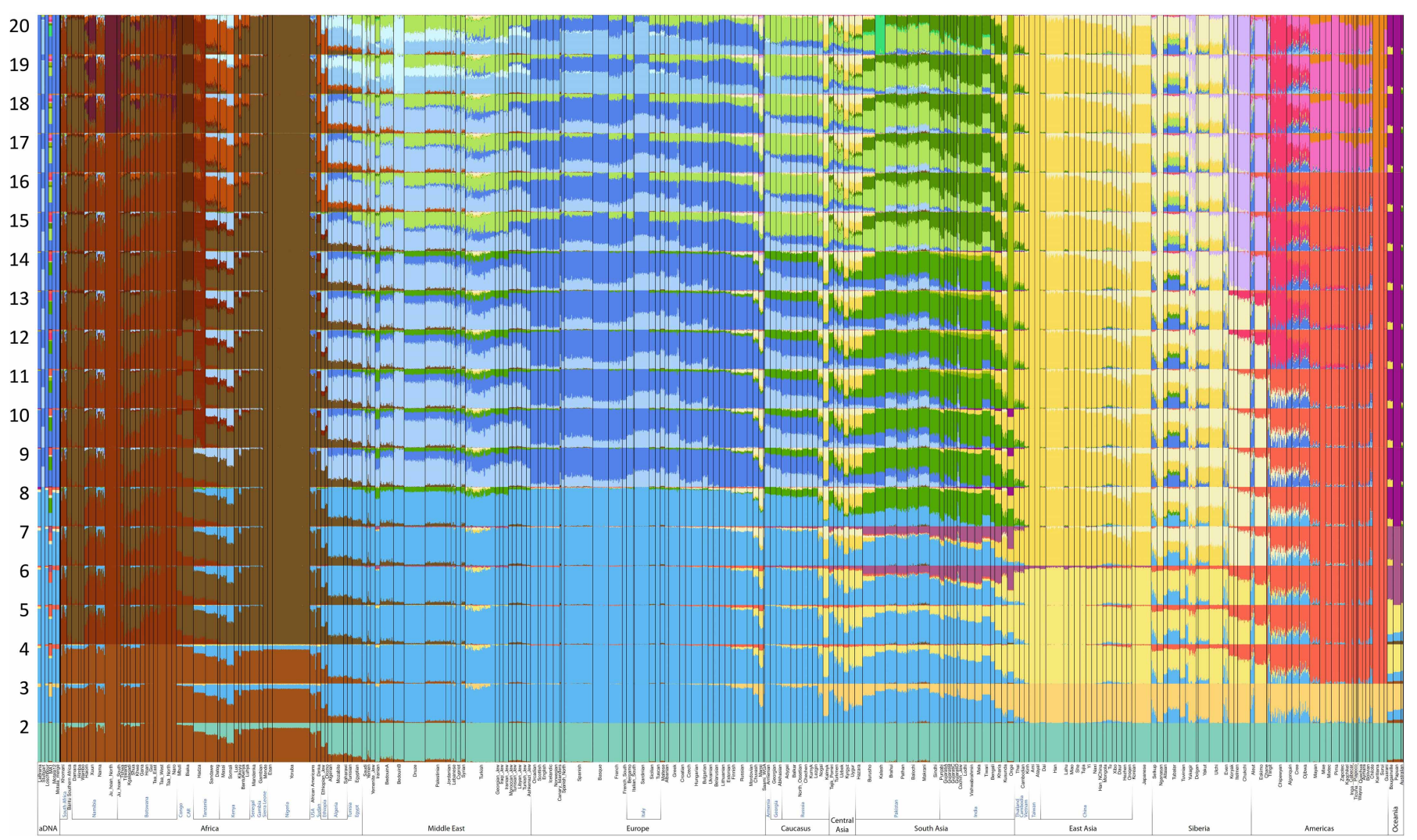

Extended Data Figure $3 \mid$ ADMIXTURE analysis $(K=\mathbf{2}$ to $K=\mathbf{2 0})$. Ancient samples (Loschbour, Stuttgart, Motala_merge, Motala12, MA1, and LaBraña) are on the left. 


\section{RESEARCH LETTER}

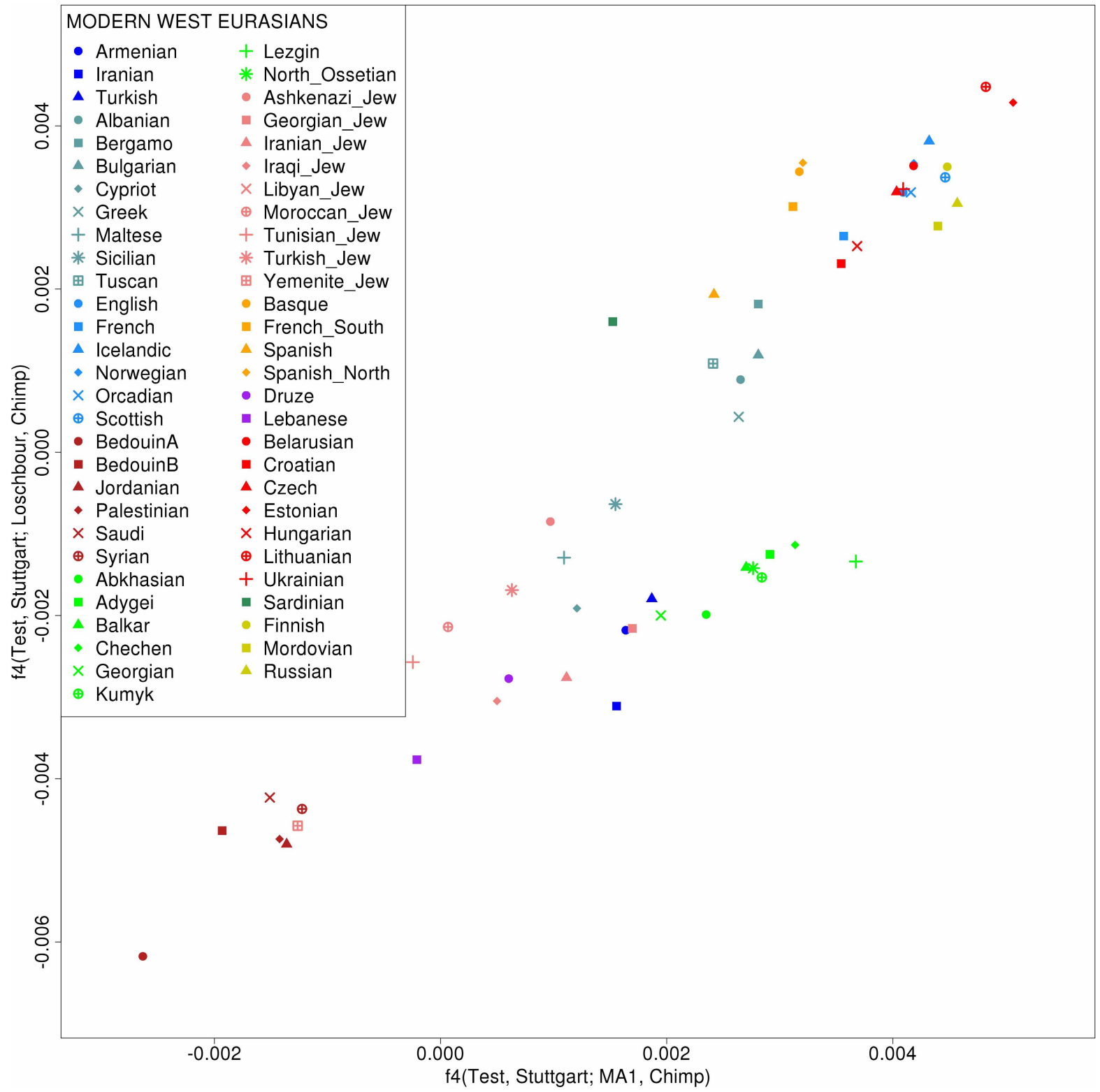

Extended Data Figure $4 \mid$ ANE ancestry is present in both Europe and the Near East but WHG ancestry is restricted to Europe, which cannot be due to a single admixture event. On the $x$ axis we present the statistic $f_{4}($ Test, Stuttgart; MA1, Chimp), which measures where MA1 shares more alleles with a test population than with Stuttgart. It is positive for most European and Near Eastern populations, consistent with ANE (MA1-related) gene flow into both regions. On the $y$ axis we present the statistic $f_{4}$ (Test, Stuttgart; Loschbour, Chimp), which measures whether Loschbour shares more alleles with a test sample than with Stuttgart. Only European populations show positive values of this statistic, providing evidence of WHG (Loschbour-related) admixture only in Europeans. 


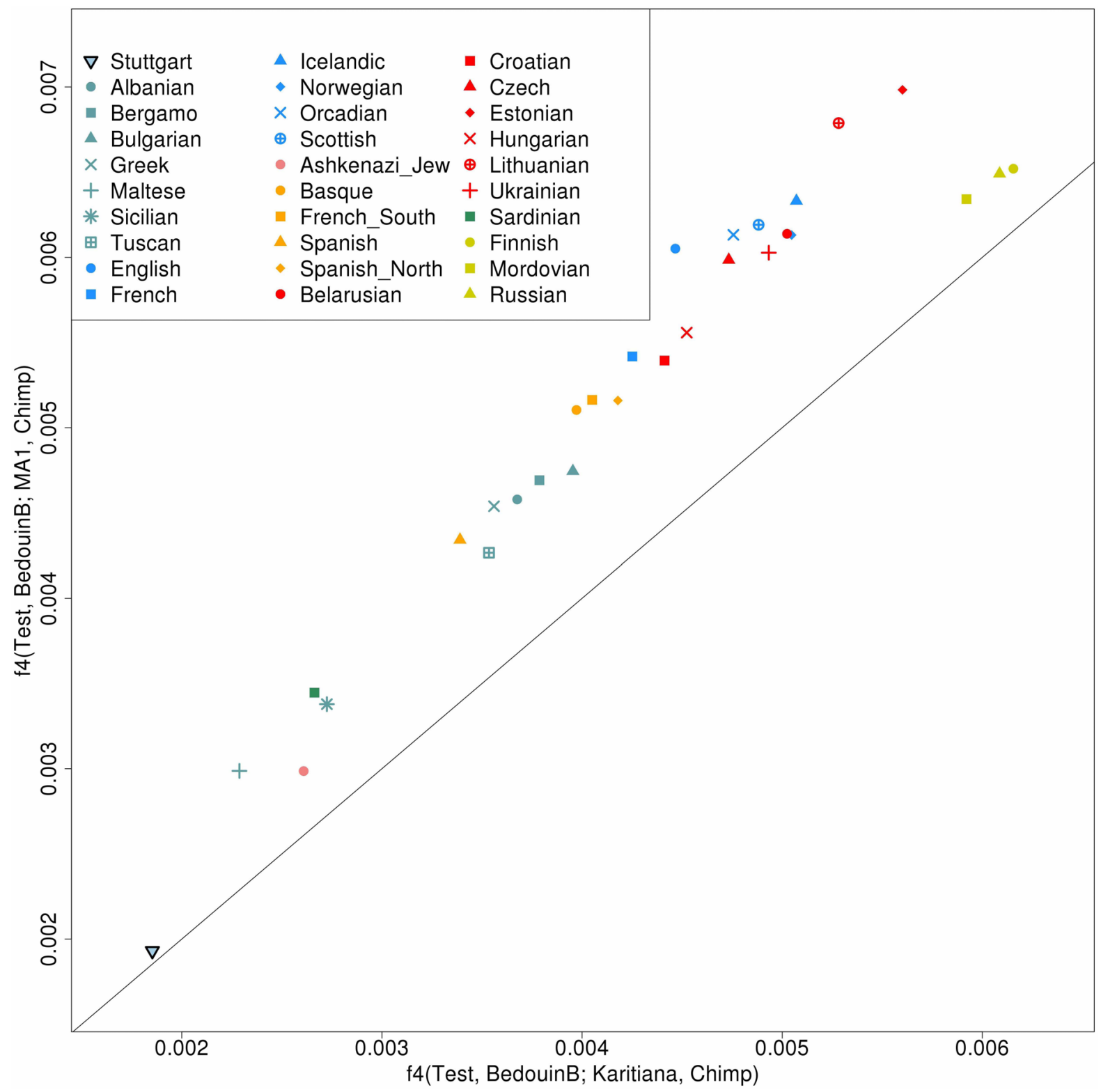

Extended Data Figure $5 \mid$ MA1 is the best surrogate for ANE for which we have data. Europeans share more alleles with MA1 than with Karitiana, as we see from the fact that in a plot of $f_{4}$ (Test, BedouinB; MA1, Chimp) and

$f_{4}$ (Test, BedouinB; Karitiana, Chimp), the European cline deviates in the direction of MA1, rather than Karitiana (the slope is $>1$ and European populations are above the line indicating inequality of these two statistics). 


\section{RESEARCH LETTER}

a

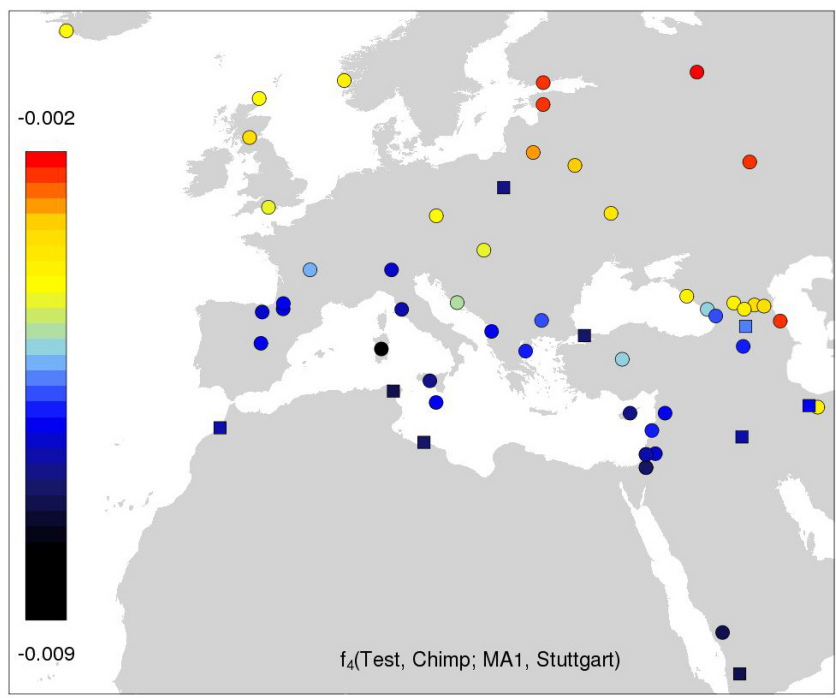

C

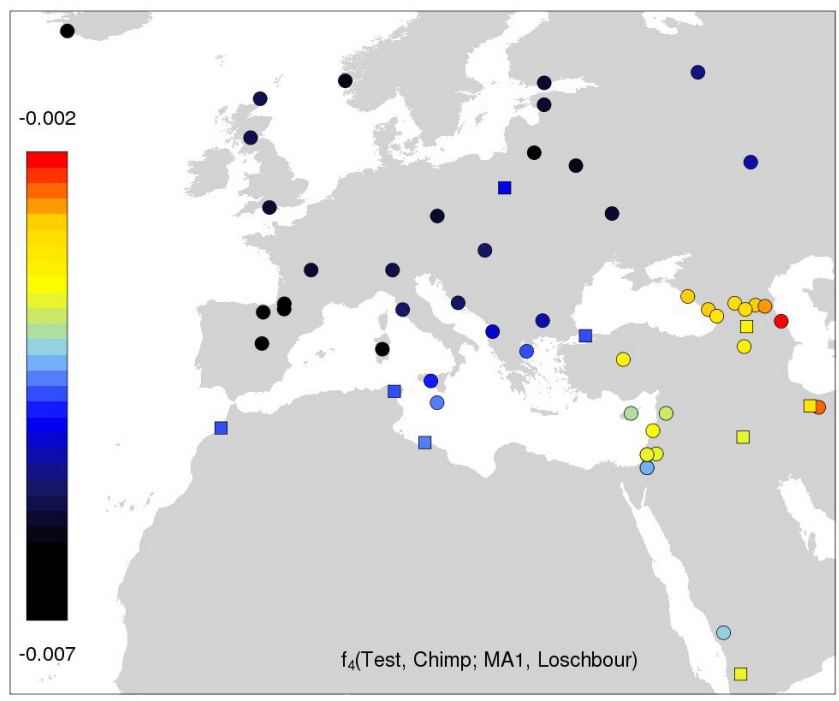

Extended Data Figure 6 The differential relatedness of west Eurasians to Stuttgart (EEF), Loschbour (WHG), and MA1 (ANE) cannot be explained by two-way mixture. We plot on a West Eurasian map the statistic $f_{4}$ (Test, Chimp; $A_{1}, A_{2}$ ), where $A_{1}$ and $A_{2}$ are a pair of the three ancient samples representing the three ancestral populations of Europe. a, In both Europe and the Near East/Caucasus, populations from the south have more relatedness to Stuttgart than those from the north where ANE influence is also important. b, Northern European populations share more alleles with Loschbour than with Stuttgart, as they have additional WHG ancestry beyond what was already b

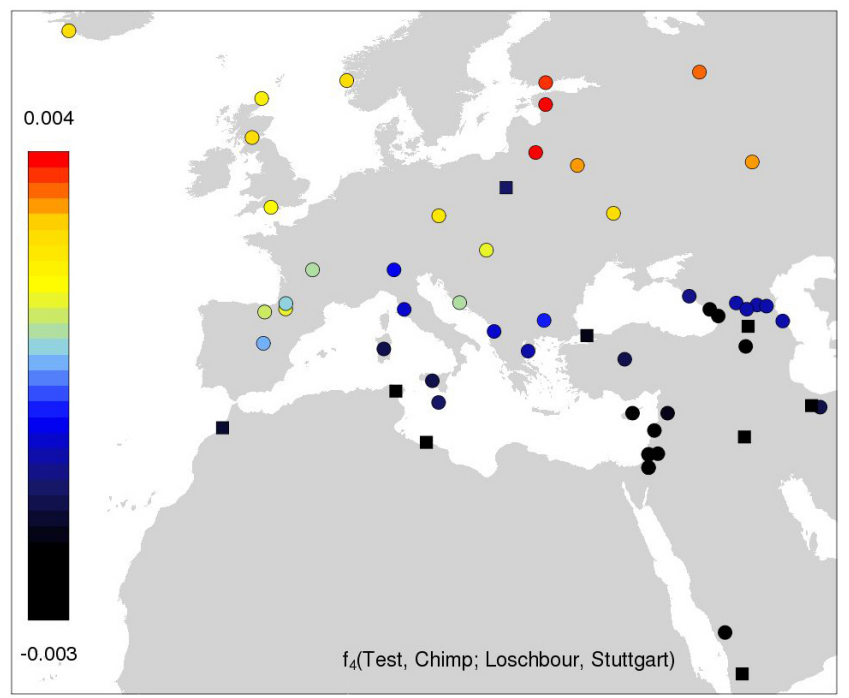

present in EEF. c, We observe a striking contrast between Europe west of the Caucasus and the Near East in degree of relatedness to WHG. In Europe, there is a much higher degree of allele sharing with Loschbour than with MA1, which we ascribe to the $60-80 \% \mathrm{WHG} /(\mathrm{WHG}+\mathrm{ANE})$ ratio in most Europeans that we report in Supplementary Information section 14. In contrast, the Near East has no appreciable WHG ancestry but some ANE ancestry, especially in the northern Caucasus. (Jewish populations are marked with a square in this figure to assist in interpretation as their ancestry is often anomalous for their geographic regions.) 


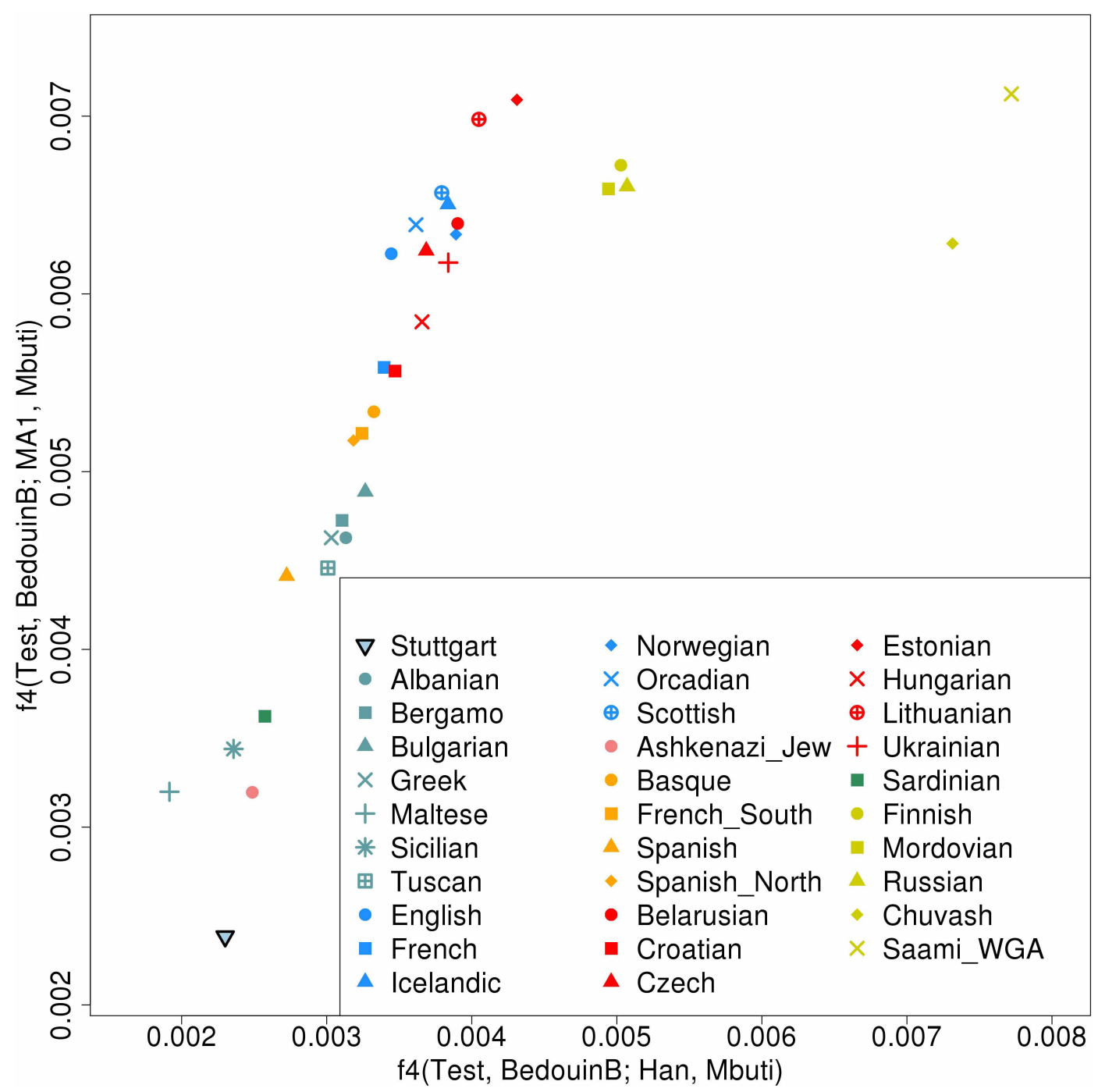

Extended Data Figure $7 \mid$ Evidence for Siberian gene flow into far north-eastern Europe. Some north-eastern European populations (Chuvash, Finnish, Russian, Mordovian, Saami) share more alleles with Han Chinese than with other Europeans who are arrayed in a cline from Stuttgart to Lithuanians/Estonians in a plot of $f_{4}$ (Test, BedouinB; Han, Mbuti) against $f_{4}$ (Test, BedouinB; MA1, Mbuti). 


\section{RESEARCH LETTER}

Extended Data Table 1 | West Eurasians genotyped on the Human Origins array and key $f$ statistics

\begin{tabular}{|c|c|c|c|c|c|c|c|c|c|c|c|c|c|c|c|c|c|c|c|c|c|c|c|c|c|c|}
\hline & \multicolumn{3}{|c|}{ Sampling Location } & \multicolumn{4}{|c|}{ Lowest $f_{3}\left(X_{;}\right.$Ref $f_{1}$ Ref $\left.f_{3}\right)$} & \multicolumn{5}{|c|}{$\begin{array}{l}\text { Lowest } f_{3}(X ; \text { EEF, WHG) } \\
(\mathrm{Z}<0 \text { and } Z \text { diff }<3 \text { reported })\end{array}$} & \multicolumn{5}{|c|}{$\begin{array}{l}\text { Lowest } f_{3}(X ; \text { N Near East, WHG) } \\
(Z<0 \text { and } Z \text { diff }<3 \text { reported })\end{array}$} & \multicolumn{5}{|c|}{$\begin{array}{l}\text { Lowest } f_{3}\left(X_{;} E E F, A N E\right) \\
(Z<0 \text { and } Z \text { diff }<3 \text { reported })\end{array}$} & \multicolumn{2}{|c|}{$\begin{array}{c}f_{f}(\text { Stuttgart, } X ; \\
\text { Loschbour, Chimp) }\end{array}$} & \multicolumn{2}{|c|}{$\begin{array}{l}f_{f}(\text { Stuttgart, } X ; \\
\text { MA1, Chimp) }\end{array}$} \\
\hline & $\mathrm{N}$ & Lat. & Long. & Ref $_{1}$ & $\mathrm{Ret}_{3}$ & statistic & $z$ & Ref $_{1}$ & $\mathrm{Ret}_{2}$ & statistic & $z$ & Zdiff & $\mathrm{Ref}_{1}$ & $\mathrm{Ref}_{2}$ & statatsitic & 2 & Zdiff & $\operatorname{Ref}_{1}$ & $\mathrm{Ret}_{3}$ & statistic & $z$ & Zdilf & statsistic & & statistic & \\
\hline $\begin{array}{l}\text { Abkhasian } \\
\text { Adygei }\end{array}$ & 99 & $\begin{array}{l}43 \\
44\end{array}$ & $\begin{array}{r}41.02 \\
39\end{array}$ & Stu & MA1 & -0.0053 & -2.9 & & & & & & Georgian & LaB & -0.0004 & -0.5 & 2.6 & Stu & MA1 & -0.0053 & -2.9 & 0.0 & 0.0020 & 4.2 & -0.0023 & -4.7 \\
\hline $\begin{array}{l}\text { Adygei } \\
\text { Alyanian }\end{array}$ & $\begin{array}{r}17 \\
6\end{array}$ & $\begin{array}{r}44 \\
41.33\end{array}$ & $\begin{array}{r}39 \\
19.83 \\
V\end{array} \mathrm{l}$ & $\begin{array}{l}\text { Piapoco } \\
\text { Sty }\end{array}$ & Stu & -0.0073 & $\begin{array}{l}-5.9 \\
-70\end{array}$ & & & & & & Jagi Jew & 100 & & & 17 & Stu & MA1 & -0.0067 & -4.1 & 0.3 & 0.0013 & 2.6 & -0.0029 & -6.0 \\
\hline Armenian & 10 & 40.19 & 44.55 & Gujaratic & Stu & $\begin{array}{l}-0.0721 \\
-0.0070\end{array}$ & -8.2 & & & & & & Iraq__Jew & Los & -0.0090 & -9.1 & 1.1 & Stu & MA1 & -0.0068 & -4.1 & 0.1 & $\begin{array}{l}-0.00092 \\
0.0022\end{array}$ & $\begin{array}{l}-1.8 \\
4.5\end{array}$ & $\begin{array}{l}-0.027 \\
-0.0016\end{array}$ & $\begin{array}{l}-5.4 \\
-3.3 \\
\text {. }\end{array}$ \\
\hline Ashkenazi_Jew & 7 & 52.23 & 21.02 & Stu & MA1 & -0.0057 & -3.4 & & & & & & Iraqi_Jew & Los & -0.0042 & -4.7 & 1.0 & Stu & MA1 & -0.0057 & -3.4 & 0.0 & 0.0008 & 1.7 & -0.0010 & -2.0 \\
\hline Balkar & 10 & 43.48 & 43.62 & Piapoco & Stu & -0.0113 & -8.9 & & & & & & & & & & & Stu & MA1 & -0.0092 & -5.5 & 1.1 & 0.0014 & 2.9 & -0.0027 & -5.6 \\
\hline Basque & 29 & 43.04 & -0.65 & Iraqi_Jew & Los & -0.0083 & -10.3 & Stu & Los & -0.0061 & -3.8 & 1.3 & Iraqi_Jew & Los & -0.0083 & -10.3 & 0.0 & Stu & MA1 & -0.0041 & -2.4 & 2.2 & -0.0034 & -7.2 & -0.0032 & -6.7 \\
\hline BedouinA & 25 & 31 & 35 & Esan & Stu & -0.0162 & -18.2 & & & & & & & & & & & & & & & & 0.0062 & 13.0 & 0.0026 & 5.4 \\
\hline BedouinB & 19 & 31 & 35 & Esan & Stu & 0.0089 & 7.8 & & & & & & & & & & & & & & & & 0.0046 & 9.3 & 0.0019 & 3.9 \\
\hline Belarusian & 10 & 53.92 & 28.01 & Georgian & Los & -0.0133 & -17.6 & & & & & & Georgian & Los & -0.0133 & -17.6 & 0.0 & Stu & MA1 & -0.0102 & -6.1 & 1.9 & -0.0035 & -6.9 & -0.0042 & -8.6 \\
\hline Bergamo & 12 & 46 & 10 & Stu & MA1 & -0.0106 & -6.2 & Stu & Los & -0.0068 & -4.2 & 1.7 & Iraqi_Jew & Los & -0.0100 & -11.9 & 0.3 & Stu & MA1 & -0.0106 & -6.2 & 0.0 & -0.0018 & -3.9 & -0.0028 & -5.8 \\
\hline Bulgarian & 10 & 42.16 & 24.74 & Stu & MA1 & -0.0130 & -8.2 & Stu & LaB & -0.0074 & -4.5 & 2.8 & Iraqi_Jew & Los & -0.0106 & -12.4 & 1.5 & Stu & MA1 & -0.0130 & -8.2 & 0.0 & -0.0012 & -2.5 & -0.0028 & -5.9 \\
\hline Chechen & 9 & 43.33 & 45.65 & Stu & MA1 & -0.0056 & -3.2 & & & & & & Georgian & Los & -0.0002 & -0.3 & 2.8 & Stu & MA1 & -0.0056 & -3.2 & 0.0 & 0.0011 & 2.3 & -0.0031 & -6.2 \\
\hline Croatian & 10 & 43.51 & 16.45 & Stu & MA1 & -0.0114 & -6.7 & Stu & Los & -0.0065 & -3.8 & 2.1 & Iraqi_Jew & Los & -0.0112 & -13.0 & 0.2 & Stu & MA1 & -0.0114 & -6.7 & 0.0 & -0.0023 & -4.7 & -0.0035 & -7.4 \\
\hline Cypriot & 8 & 35.13 & 33.43 & Stu & MA1 & -0.0057 & -3.2 & & & & & & Yemenite_Jew & Los & -0.0013 & -1.5 & 2.5 & Stu & MA1 & -0.0057 & -3.2 & 0.0 & 0.0019 & 3.9 & -0.0012 & -2.5 \\
\hline $\begin{array}{l}\text { Czech } \\
\text { D }\end{array}$ & $\begin{array}{l}10 \\
39\end{array}$ & $\begin{array}{r}50.1 \\
32\end{array}$ & $\begin{array}{r}14.4 \\
35\end{array}$ & Georgian & Los & -0.0137 & -17.9 & Stu & Los & -0.0088 & -5.3 & 3.0 & Georgian & Los & -0.0137 & -17.9 & 0.0 & Stu & MA1 & -0.0121 & -7.2 & 0.9 & -0.0032 & -6.6 & -0.0040 & -8.2 \\
\hline $\begin{array}{l}\text { Druze } \\
\text { English }\end{array}$ & $\begin{array}{l}39 \\
10\end{array}$ & $\begin{array}{r}32 \\
5075\end{array}$ & $\begin{array}{r}35 \\
-209 \\
-209\end{array}$ & Stu & MA1 & -0.0024 & $\begin{array}{r}-1.5 \\
-148\end{array}$ & $S_{1}$ & 10 & ๑ Р००९० & 55 & $2 ?$ & Jaci Jave & & & & & Stu & MA1 & -0.0024 & -1.5 & 0.0 & 0.0028 & 5.9 & -0.0006 & -1.3 \\
\hline $\begin{array}{l}\text { English } \\
\text { Estonian }\end{array}$ & 10 & 58.54 & $\begin{array}{l}-2.099 \\
24.89\end{array}$ & $\begin{array}{l}\text { Iraqu_Jew } \\
\text { Abkhasian }\end{array}$ & $\begin{array}{l}\text { Los } \\
\text { Los }\end{array}$ & $\begin{array}{l}-0.0129 \\
-0.0124\end{array}$ & $\begin{array}{l}-14.8 \\
-15.1\end{array}$ & Stu & Los & -0.0090 & -5.5 & 2.2 & $\begin{array}{l}\text { Aracl_ew } \\
\text { Abkhasian }\end{array}$ & $\begin{array}{l}\text { Los } \\
\text { Los }\end{array}$ & $\begin{array}{l}-0.0129 \\
-0.0124\end{array}$ & $\begin{array}{l}-14.8 \\
-15.1\end{array}$ & $\begin{array}{l}0.0 \\
0.0\end{array}$ & $\begin{array}{l}\text { Stu } \\
\text { Stu }\end{array}$ & MA1 & $\begin{array}{l}-0.0125 \\
-0.0094\end{array}$ & $\begin{array}{l}-7.4 \\
-5.6\end{array}$ & $\begin{array}{l}0.1 \\
1.9\end{array}$ & $\begin{array}{l}-0.0032 \\
-0.0043\end{array}$ & $\begin{array}{l}-6.5 \\
-8.5\end{array}$ & $\begin{array}{l}-0.0041 \\
-0.0051\end{array}$ & $\begin{array}{r}-8.5 \\
-10.1\end{array}$ \\
\hline Finnish & 7 & 60.2 & 24.9 & Abkhasian & Los & -0.0102 & -11.3 & & & & & & Abkhasian & Los & -0.0102 & -11.3 & 0.0 & Stu & MA1 & -0.0078 & -4.4 & 1.4 & -0.0035 & -6.9 & -0.0045 & -9.1 \\
\hline French & 25 & 46 & 2 & Stu & MA1 & -0.0131 & -8.4 & Stu & Los & -0.0098 & -6.3 & 1.5 & Iraqi_Jew & Los & -0.0129 & -16.8 & 0.2 & Stu & MA1 & -0.0131 & -8.4 & 0.0 & -0.0027 & -5.6 & -0.0036 & -7.7 \\
\hline French_South & 7 & 43.44 & -0.62 & Iraqi_Jew & Los & -0.0095 & -9.5 & Stu & $\mathrm{LaB}$ & -0.0089 & -5.0 & 0.3 & Iraqi_Jew & Los & -0.0095 & -9.5 & 0.0 & Stu & MA1 & -0.0086 & -4.8 & 0.4 & $\begin{array}{l}-0.0030 \\
\end{array}$ & -6.2 & -0.0031 & -6.2 \\
\hline Georgian & 10 & 42.5 & 41.85 & Gujaratic & Stu & -0.0036 & -4.0 & & & & & & & & & & & Stu & MA1 & -0.0036 & -2.1 & -0.2 & 0.0020 & 4.2 & -0.0019 & -3.9 \\
\hline Georgian_Jew & 7 & 41.72 & 44.78 & Gujaratic & Stu & -0.0009 & -0.9 & & & & & & & & & & & Stu & MA1 & -0.0002 & -0.1 & 0.3 & 0.0022 & 4.3 & -0.0017 & -3.4 \\
\hline Greek & 20 & 39.84 & 23.17 & Stu & MA1 & -0.0118 & -7.4 & & & & & & Iraqi_Jew & Los & -0.0080 & -11.1 & 2.3 & Stu & MA1 & -0.0118 & -7.4 & 0.0 & -0.0004 & -0.9 & -0.0026 & -5.6 \\
\hline Hungarian & 20 & 47.49 & 19.08 & Stu & MA1 & -0.0133 & -8.4 & Stu & Los & -0.0087 & -5.6 & 2.2 & Iraqi__Jew & Los & -0.0127 & -15.9 & 0.4 & Stu & MA1 & -0.0133 & -8.4 & 0.0 & -0.0025 & -5.3 & -0.0037 & -7.8 \\
\hline Icelandic & 12 & 64.13 & -21.93 & Abkhasian & Los & -0.0121 & -15.6 & Stu & Los & -0.0078 & -4.8 & 2.7 & Abkhasian & Los & -0.0121 & -15.6 & 0.0 & Stu & MA1 & -0.0097 & -5.9 & 1.5 & -0.0038 & -7.7 & -0.0043 & -8.9 \\
\hline Iranian & 8 & 35.59 & 51.46 & Plapoco & Stu & -0.0094 & -7.2 & & & & & & & & & & & Stu & MA1 & -0.0087 & -5.2 & 0.4 & 0.0031 & 6.3 & -0.0016 & -3.2 \\
\hline Iranian_Jew & 9 & $\begin{array}{r}35.7 \\
33.33\end{array}$ & 51.42 & Gujaratic & Stu & -0.0018 & -2.0 & & & & & & & & & & & Stu & MA1 & -0.0012 & -0.6 & 0.2 & 0.0028 & 5.7 & -0.0011 & -2.2 \\
\hline Iraqi_Jew & 6 & 33.33 & $\begin{array}{l}44.42 \\
35.91\end{array}$ & Vishwabrahmin & Stu & -0.0026 & -2.6 & & & & & & & & & & & Stu & MA1 & -0.0009 & -0.5 & 0.9 & 0.0030 & 6.1 & -0.0005 & -1.0 \\
\hline Jordanian & 9 & $\begin{array}{l}32.05 \\
43.25\end{array}$ & $\begin{array}{r}35.91 \\
44.58\end{array}$ & Esan & $\begin{array}{l}\text { Stu } \\
\text { Stu }\end{array}$ & -0.0145 & -14.3 & & & & & & & & & & & & & & & & 0.0048 & 9.6 & 0.0014 & 2.8 \\
\hline $\begin{array}{l}\text { Kumyk } \\
\text { Lebanese }\end{array}$ & $\begin{array}{l}8 \\
8\end{array}$ & $\begin{array}{l}43.25 \\
3382\end{array}$ & $\begin{array}{l}46.58 \\
3557\end{array}$ & $\begin{array}{l}\text { Piapoco } \\
\text { Fsan }\end{array}$ & $\begin{array}{l}\text { Stu } \\
\text { Stut }\end{array}$ & -0.0111 & -8.2 & & & & & & & & & & & $\begin{array}{l}\text { Stu } \\
\text { Stutu }\end{array}$ & MA1 & -0.0109 & -6.5 & 0.1 & 0.0015 & 3.1 & -0.0028 & -5.7 \\
\hline $\begin{array}{l}\text { Lebanese } \\
\text { Lezgin }\end{array}$ & $\begin{array}{l}8 \\
9\end{array}$ & $\begin{array}{l}33.82 \\
42.12\end{array}$ & $\begin{array}{l}35.57 \\
48.18\end{array}$ & $\begin{array}{l}\text { Esan } \\
\text { Stu }\end{array}$ & $\begin{array}{l}\text { Stu } \\
\text { MA1 }\end{array}$ & $\begin{array}{l}-0.0105 \\
-0.0100\end{array}$ & $\begin{array}{l}-9.4 \\
-60\end{array}$ & & & & & & & & & & & $\begin{array}{l}\text { Stu } \\
\text { Stu }\end{array}$ & $\begin{array}{l}\text { MA1 } \\
\text { MA1 }\end{array}$ & $\begin{array}{l}-0.0068 \\
-0.0100\end{array}$ & $\begin{array}{l}-3.9 \\
-6.0\end{array}$ & 1.9 & $\begin{array}{l}0.0038 \\
0.0013\end{array}$ & 7.7 & 0.0002 & 0.4 \\
\hline Libyan_Jew & 9 & 32.92 & $\begin{array}{l}13.18 \\
\end{array}$ & Esan & Stu & $\begin{array}{l}-0.0051 \\
-0.0051\end{array}$ & -4.4 & & & & & & & & & & & $\begin{array}{l}\text { Stu } \\
\text { Stu }\end{array}$ & MA1 & 0.0000 & 0.0 & 2.7 & 0.0030 & $\begin{array}{l}.2 .1 \\
6.2\end{array}$ & 0.0004 & $\begin{array}{l}-1.5 \\
0.9\end{array}$ \\
\hline Lithuanian & 10 & 54.9 & 23.92 & Abkhasian & Los & -0.0119 & -14.9 & & & & & & Abkhasian & Los & -0.0119 & -14.9 & 0.0 & Stu & MA1 & -0.0069 & -3.9 & 2.8 & -0.0045 & -9.0 & -0.0048 & -9.9 \\
\hline Maltese & 8 & 35.94 & 14.38 & Stu & MA1 & -0.0086 & -4.9 & & & & & & Yemenite_Jew & Los & -0.0051 & -6.0 & 2.0 & Stu & MA1 & -0.0086 & -4.9 & 0.0 & 0.0013 & 2.7 & -0.0011 & -2.3 \\
\hline Mordovian & 10 & 54.18 & 45.18 & Abkhasian & Los & -0.01 & -14.4 & & & & & & Abkhasian & Los & -0.0115 & -14.4 & 0.0 & Stu & MA1 & -0.0113 & -6.6 & 0.3 & -0.00 & -5.5 & -0.0044 & -9.0 \\
\hline Moroccan_Jew & 6 & 34.02 & -6.84 & Esan & Stu & -0.0062 & -5.2 & & & & & & Yemenite_Jew & Los & -0.0021 & -2.2 & 2.9 & Stu & MA1 & -0.0032 & -1.7 & 1.4 & 0.00 & 4.3 & -0.0001 & -0.1 \\
\hline North_Ossetian & 10 & 43.02 & 44.65 & Piapoco & Stu & -0.00 & -7.2 & & & & & & & & & & & Stu & MA1 & -0.0076 & -4.4 & 1.0 & & 2.9 & -0.0028 & -5.6 \\
\hline Norwegian & 11 & 60.36 & 5.36 & Georgian & Los & -0.0 & -14.8 & & & & & & Georgian & Los & -0.0120 & -14.8 & 0.0 & Stu & MA1 & -0.0093 & -5.4 & 1.4 & & -7.3 & -0.0042 & -8.7 \\
\hline Orcadian & 13 & 59 & -3 & Armenian & Los & -0.01 & -13.4 & Stu & Los & -0.0059 & -3.6 & 2.5 & Armenian & Los & -0.0102 & -13.4 & 0.0 & Stu & MA1 & -0.0098 & -5.9 & 0.5 & & -6.7 & -0.0042 & -8.6 \\
\hline Palestinian & 38 & 32 & 35 & Esan & Stu & -0.01 & -13.2 & & & & & & & & & & & & & & & & & 10.2 & & 3.1 \\
\hline Russiar & 22 & 61 & 40 & Chukchi & Los & -0.0119 & -11.3 & & & & & & Abkhasian & Los & -0.0119 & -17.1 & 0.0 & Stu & MA1 & -0.0106 & -6.6 & 0.8 & & -6.2 & -0.0046 & -9.4 \\
\hline $\begin{array}{l}\text { Sardidinan } \\
\text { Saudi }\end{array}$ & $\begin{array}{r}27 \\
8\end{array}$ & $\begin{array}{r}40 \\
18.49\end{array}$ & $\begin{array}{r}9 \\
42.52\end{array}$ & $\begin{array}{l}\text { Stu } \\
\text { Kgalagadi }\end{array}$ & $\begin{array}{l}\text { LaB } \\
\text { Stu }\end{array}$ & $\begin{array}{l}-0.00044 \\
-0.0042\end{array}$ & $\begin{array}{l}-2.6 \\
-3.6\end{array}$ & Stu & $\mathrm{LaB}$ & -0.0044 & -2.6 & 0.0 & Iraqui_Jew & Los & -0.0033 & -4.2 & 0.0 & Stu & MA1 & -0.0035 & -2.1 & 0.3 & $\begin{array}{r}-0.0016 \\
0.0042\end{array}$ & $\begin{array}{r}-3.4 \\
8.6\end{array}$ & $\begin{array}{l}-0.0015 \\
0.0015\end{array}$ & $\begin{array}{l}-3.3 \\
3.1 \\
\end{array}$ \\
\hline Scottish & 4 & 56.04 & -3.94 & Iraqi_Jew & Los & -0.0103 & -8.3 & & & & & & Iraqi_Jew & Los & -0.0103 & -8.3 & 0.0 & Stu & MA1 & -0.0090 & -4.7 & 0.7 & -0.0034 & -6.4 & -0.0045 & -8.7 \\
\hline Sicilian & 11 & 37.59 & 13.77 & Stu & MA1 & -0.0108 & -6.5 & & & & & & Yemenite_Jew & Los & -0.0066 & -8.1 & 2.4 & Stu & MA1 & -0.0108 & -6.5 & 0.0 & 0.0006 & 1.3 & -0.0015 & -3.2 \\
\hline Spanish & 53 & 40.43 & -2.83 & ai_Jew & Los & -0.0126 & -17.8 & Stu & Los & -0.0104 & -6.8 & 1.4 & Iraqi_Jew & Los & -0.0126 & -17.8 & 0.0 & Stu & MA1 & -0.0120 & -7.6 & 0.3 & -0.0019 & -4.2 & -0.0024 & -5.2 \\
\hline Spanish_North & 5 & 42.8 & -2.7 & Iraqi_Jew & Los & -0.0112 & -9.9 & Stu & Los & -0.0102 & -5.4 & 0.5 & Iraqi_Jew & Los & -0.0112 & -9.9 & 0.0 & Stu & MA1 & -0.0082 & -4.4 & 1.3 & -0.0035 & -6.9 & -0.0032 & -6.4 \\
\hline & 8 & 35.13 & 36.8 & & Stu & -0.0101 & -8.7 & & & & & & 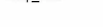 & & & & & & & & & & 0.0044 & 8.6 & 0.0012 & 2.4 \\
\hline an_Jew & 7 & 36.8 & 10. & & Stu & -0.00 & -2.0 & & & & & & & & & & & & & & & & & 5.2 & 0.0002 & 0.5 \\
\hline Turk & 56 & 39.22 & 32.66 & Piapoco & Stu & -0.0 & -11.3 & & & & & & & & & & & Stu & MA1 & -0.01 & -6.9 & 1.3 & & 3.8 & -0.0019 & -4.0 \\
\hline h_Jew & 8 & 41.02 & 28.95 & Sth & MA1 & -0.0 & -4.3 & & & & & & Yemenite_Jew & Los & -0.0049 & -5.8 & 1.4 & Stu & 1 & -0.0075 & -4.3 & 0.0 & 0.00 & 3.6 & -0.0006 & -1.3 \\
\hline Tuscan & 8 & 43 & 11 & Stu & MA1 & -0.0109 & -6.4 & Stu & Los & -0.0055 & -3.2 & 2.3 & Iraqi_Jew & Los & -0.0092 & -10.1 & 0.9 & Stu & MA1 & -0.0109 & -6.4 & 0.0 & & -2.2 & -0.0024 & -5.0 \\
\hline $\begin{array}{l}\text { Ukrainian } \\
\text { Yemenite Jew }\end{array}$ & $\begin{array}{l}9 \\
8\end{array}$ & $\begin{array}{l}50.29 \\
15.35\end{array}$ & $\begin{array}{r}31.56 \\
44.2\end{array}$ & $\begin{array}{l}\text { Georgian } \\
\text { Esan }\end{array}$ & $\begin{array}{l}\text { Los } \\
\text { Stu }\end{array}$ & $\begin{array}{l}-0.0134 \\
-0.0227\end{array}$ & $\begin{array}{l}-16.7 \\
-2.4\end{array}$ & & & & & & Georgian & Los & -0.0134 & -16.7 & 0.0 & Stu & MA1 & -0.0114 & -6.6 & 1.3 & $\begin{array}{l}-0.0032 \\
0.0046\end{array}$ & $\begin{array}{l}-6.4 \\
9.1\end{array}$ & $\begin{array}{l}-0.0041 \\
0.0013\end{array}$ & $\begin{aligned}-8.5 \\
2.6\end{aligned}$ \\
\hline & & & & & & & & & & & & & & & & & & & & & & & & & & 2.6 \\
\hline
\end{tabular}

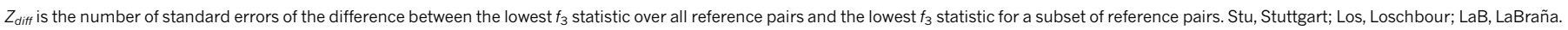


Extended Data Table 2 | Confirmation of key findings on transversions and on whole-genome sequence data

\begin{tabular}{|c|c|c|c|c|c|c|c|c|c|c|c|c|c|c|}
\hline \multirow{2}{*}{ Interpretation } & \multicolumn{8}{|c|}{$D(A, B ; C, D)$ on Human Origins genotype data } & \multicolumn{6}{|c|}{$D(A, B ; C, D)$ on whole genome sequence data transversions } \\
\hline & A & B & c & D & $\begin{array}{l}594,924 \mathrm{~S} \\
\text { statistic }\end{array}$ & $\begin{array}{l}\text { Ps } \\
z\end{array}$ & $\begin{array}{l}110,817 \text { transve } \\
\text { statistic }\end{array}$ & $\begin{array}{l}\text { ersions } \\
\mathrm{Z}\end{array}$ & A & B & c & D & statistic & z \\
\hline Stuttgart has Near Eastern ancestry & Stuttgart & Armenian & Loschbour & Chimp & 0.0219 & 4.5 & 0.0189 & 2.9 & & & & & & \\
\hline \multirow{2}{*}{$\begin{array}{l}\text { Europeans have more WHG-related ancestry than } \\
\text { Stuttgart }\end{array}$} & Stuttgart & French & Loschbour & Chimp & -0.0266 & -5.7 & -0.031 & -5.0 & Stuttgart & French2 & Loschbour & Chimp & -0.03 & -4.7 \\
\hline & Lithuanian & Stuttgart & Loschbour & Chimp & 0.0446 & 9.1 & 0.0477 & 7.2 & & & & & & \\
\hline \multirow{2}{*}{$\begin{array}{l}\text { West Eurasians have more ANE-related ancestry } \\
\text { than Stuttgart }\end{array}$} & French & Stuttgart & MA1 & Chimp & 0.0367 & 7.7 & 0.0386 & 5.5 & French2 & Stuttgart & MA1 & Chimp & 0.037 & 6.4 \\
\hline & Lezgin & Stuttgart & MA1 & Chimp & 0.0372 & 7.6 & 0.0409 & 5.6 & & & & & & \\
\hline $\begin{array}{c}\text { MA1 is a better surrogate of ANE ancestry than } \\
\text { Karitiana }\end{array}$ & French & Chimp & MA1 & Karitiana & 0.0207 & 4.5 & 0.0214 & 2.8 & French2 & Chimp & MA1 & Karitiana2 & 0.026 & 3.8 \\
\hline \multirow{9}{*}{$\begin{array}{l}\text { Eastern non-Africans closer to WHG/ANE/SHG } \\
\text { than to EEF }\end{array}$} & Loschbour & Stuttgart & Onge & Chimp & 0.0196 & 3.5 & 0.0202 & 2.5 & & & & & & \\
\hline & Loschbour & Stuttgart & Papuan & Chimp & 0.0142 & 2.6 & 0.0127 & 1.5 & Loschbour & Stuttgart & Papuan2 & Chimp & 0.017 & 2.7 \\
\hline & Loschbour & Stuttgart & Dai & Chimp & 0.0164 & 3.2 & 0.021 & 2.8 & Loschbour & Stuttgart & Dai2 & Chimp & 0.018 & 2.9 \\
\hline & MA1 & Stuttgart & Papuan & Chimp & 0.0139 & 2.2 & 0.0103 & 1.0 & MA1 & Stuttgart & Papuan2 & Chimp & 0.018 & 2.8 \\
\hline & MA1 & Stuttgart & Dai & Chimp & 0.0174 & 3.0 & 0.016 & 1.7 & MA1 & Stuttgart & Dai2 & Chimp & 0.028 & 4.3 \\
\hline & Motala12 & Stuttgart & Papuan & Chimp & 0.0182 & 3.2 & 0.011 & 1.1 & Motala12 & Stuttgart & Papuan2 & Chimp & 0.023 & 3.7 \\
\hline & Motala12 & Stuttgart & Dai & Chimp & 0.0156 & 2.8 & 0.0149 & 1.6 & Motala12 & Stuttgart & Dai2 & Chimp & 0.02 & 3.2 \\
\hline & LaBrana & Stuttgart & Papuan & Chimp & 0.0123 & 2.3 & 0.0101 & 1.1 & LaBrana & Stuttgart & Papuan2 & Chimp & 0.02 & 3.2 \\
\hline & LaBrana & Stuttgart & Dai & Chimp & 0.0149 & 2.9 & 0.0228 & 2.5 & LaBrana & Stuttgart & Dai2 & Chimp & 0.024 & 3.7 \\
\hline Native Americans closer to ANE than to WHG & Karitiana & Chimp & MA1 & Loschbour & 0.0467 & 7.1 & 0.0467 & 4.4 & Karitiana2 & Chimp & MA1 & Loschbour & 0.052 & 7.1 \\
\hline \multirow{2}{*}{$\begin{array}{l}\text { West Eurasians closer to Native Americans than to } \\
\text { other Eastern non-Africans }\end{array}$} & Stuttgart & Chimp & Karitiana & Papuan & 0.0559 & 10.9 & 0.0474 & 6.6 & Stuttgart & Chimp & Karitiana2 & Papuan2 & 0.052 & 7.6 \\
\hline & Stuttgart & Chimp & Karitiana & Onge & 0.0237 & 5.1 & 0.0179 & 2.6 & & & & & & \\
\hline \multirow{6}{*}{$\begin{array}{l}\text { Ancient Eurasian hunter-gatherers equally related } \\
\text { to Eastern non-Africans other than Native } \\
\text { Americans }\end{array}$} & Loschbour & MA1 & Dai & Chimp & -0.0015 & -0.2 & 0.0016 & 0.2 & Loschbour & MA1 & Dai2 & Chimp & -0.013 & -1.9 \\
\hline & Loschbour & MA1 & Papuan & Chimp & 0.0002 & 0.0 & 0.0012 & 0.1 & Loschbour & MA1 & Papuan2 & Chimp & -0.003 & -0.4 \\
\hline & Loschbour & Motala12 & Dai & Chimp & 0.0024 & 0.4 & 0.009 & 0.9 & Loschbour & Motala12 & Dai2 & Chimp & -0.002 & -0.3 \\
\hline & Loschbour & Motala12 & Papuan & Chimp & -0.0028 & -0.4 & 0.0046 & 0.5 & Loschbour & Motala12 & Papuan2 & Chimp & -0.004 & -0.6 \\
\hline & MA1 & Motala12 & Dai & Chimp & 0.0026 & 0.4 & 0.0047 & 0.4 & MA1 & Motala12 & Dai2 & Chimp & 0.01 & 1.5 \\
\hline & MA1 & Motala12 & Papuan & Chimp & -0.0047 & -0.7 & -0.001 & -0.1 & MA1 & Motala12 & Papuan2 & Chimp & -0.004 & -0.5 \\
\hline \multirow{3}{*}{ LaBrana and Loschbour are a clade } & LaBrana & Loschbour & Dai & Chimp & -0.0028 & -0.5 & 0.0024 & 0.3 & LaBrana & Loschbour & Dai2 & Chimp & 0.007 & 1.1 \\
\hline & LaBrana & Loschbour & Papuan & Chimp & -0.0031 & -0.5 & -0.0012 & -0.1 & LaBrana & Loschbour & Papuan2 & Chimp & 0.002 & 0.3 \\
\hline & LaBrana & Loschbour & MA1 & Chimp & -0.006 & -0.8 & 0.0101 & 0.7 & LaBrana & Loschbour & MA1 & Chimp & 0.005 & 0.7 \\
\hline \multirow{2}{*}{ SHG closer to ANE than to WHG } & Motala12 & Loschbour & MA1 & Chimp & 0.0425 & 5.3 & 0.0353 & 2.6 & Motala12 & Loschbour & MA1 & Chimp & 0.042 & 5.9 \\
\hline & Motala12 & LaBrana & MA1 & Chimp & 0.0465 & 5.8 & 0.0347 & 2.4 & Motala12 & LaBrana & MA1 & Chimp & 0.038 & 5.4 \\
\hline LaBrana and Loschbour equally related to Stuttgart & LaBrana & Loschbour & Stuttgart & Chimp & -0.0176 & -2.6 & -0.0106 & -1.0 & LaBrana & Loschbour & Stuttgart & Chimp & -0.012 & -1.8 \\
\hline
\end{tabular}




\section{RESEARCH LETTER}

Extended Data Table 3 | Admixture proportions for European populations

\begin{tabular}{|c|c|c|c|c|c|c|c|c|c|c|c|c|c|c|c|}
\hline & \multicolumn{3}{|c|}{$\begin{array}{l}\text { Full modeling of } \\
\text { population relationships } \\
\text { (individual fits) }\end{array}$} & \multicolumn{6}{|c|}{$\begin{array}{l}\text { Full modeling of } \\
\text { population relationships } \\
\text { (averaged fits) }\end{array}$} & \multicolumn{3}{|c|}{$\begin{array}{l}\text { Modeling of population } \\
\text { relationships with } \\
\text { minimal assumptions }\end{array}$} & \multicolumn{3}{|c|}{$\begin{array}{l}\text { Model-based (averaged) } \\
\text { - Model with minimal } \\
\text { assumptions (Z-score) }\end{array}$} \\
\hline & \multirow[t]{2}{*}{ EEF } & \multirow[t]{2}{*}{ WHG } & \multirow[t]{2}{*}{ ANE } & \multicolumn{2}{|r|}{ EEF } & \multicolumn{2}{|c|}{ WHG } & \multicolumn{2}{|r|}{ ANE } & \multirow[t]{2}{*}{ EEF } & \multirow[t]{2}{*}{ WHG } & \multirow[t]{2}{*}{ ANE } & \multirow[t]{2}{*}{ EEF } & \multirow[t]{2}{*}{ WHG } & \multirow[t]{2}{*}{ ANE } \\
\hline & & & & Mean & Range & Mean & Range & Mean & Range & & & & & & \\
\hline Albanian & 0.781 & 0.092 & 0.127 & 0.781 & $0.772-0.819$ & 0.082 & $0.032-0.098$ & 0.137 & $0.129-0.158$ & $0.595 \pm 0.112$ & $0.353 \pm 0.150$ & $0.052 \pm 0.049$ & 1.658 & -1.807 & 1.741 \\
\hline Ashkenazi_Jew & 0.931 & 0 & 0.069 & & & & & & & $0.938 \pm 0.146$ & $-0.021 \pm 0.185$ & $0.083 \pm 0.049$ & & & \\
\hline Basque & 0.593 & 0.293 & 0.114 & 0.569 & $0.527-0.616$ & 0.335 & $0.255-0.392$ & 0.096 & $0.076-0.129$ & $0.569 \pm 0.091$ & $0.315 \pm 0.124$ & $0.115 \pm 0.041$ & -0.001 & 0.165 & -0.472 \\
\hline Belarusian & 0.418 & 0.431 & 0.151 & 0.426 & $0.397-0.464$ & 0.408 & $0.338-0.443$ & 0.167 & $0.150-0.199$ & $0.272 \pm 0.094$ & $0.554 \pm 0.131$ & $0.174 \pm 0.047$ & 1.637 & -1.118 & -0.158 \\
\hline Bergamo & 0.715 & 0.177 & 0.108 & 0.721 & $0.704-0.793$ & 0.163 & $0.061-0.189$ & 0.117 & $0.104-0.147$ & $0.644 \pm 0.125$ & $0.248 \pm 0.170$ & $0.108 \pm 0.053$ & 0.615 & -0.503 & 0.162 \\
\hline Bulgarian & 0.712 & 0.147 & 0.141 & 0.718 & $0.707-0.778$ & 0.132 & $0.047-0.151$ & 0.151 & $0.138-0.175$ & $0.556 \pm 0.110$ & $0.328 \pm 0.143$ & $0.116 \pm 0.043$ & 1.469 & -1.372 & 0.804 \\
\hline Croatian & 0.561 & 0.293 & 0.145 & 0.564 & $0.548-0.586$ & 0.285 & $0.242-0.310$ & 0.151 & $0.137-0.172$ & $0.453 \pm 0.122$ & $0.407 \pm 0.159$ & $0.140 \pm 0.046$ & 0.911 & -0.768 & 0.238 \\
\hline Czech & 0.495 & 0.338 & 0.167 & 0.489 & $0.460-0.531$ & 0.348 & $0.273-0.382$ & 0.163 & $0.145-0.196$ & $0.402 \pm 0.117$ & $0.400 \pm 0.162$ & $0.198 \pm 0.050$ & 0.744 & -0.322 & -0.698 \\
\hline English & 0.495 & 0.364 & 0.141 & 0.503 & $0.476-0.536$ & 0.353 & $0.296-0.382$ & 0.144 & $0.130-0.169$ & $0.475 \pm 0.091$ & $0.357 \pm 0.125$ & $0.168 \pm 0.043$ & 0.304 & -0.028 & -0.561 \\
\hline Estonian & 0.322 & 0.495 & 0.183 & 0.323 & $0.293-0.345$ & 0.49 & $0.451-0.520$ & 0.187 & $0.172-0.205$ & $0.072 \pm 0.121$ & $0.778 \pm 0.176$ & $0.150 \pm 0.064$ & 2.070 & -1.636 & 0.584 \\
\hline French & 0.554 & 0.311 & 0.135 & 0.563 & $0.537-0.601$ & 0.297 & $0.230-0.328$ & 0.14 & $0.126-0.169$ & $0.498 \pm 0.097$ & $0.359 \pm 0.127$ & $0.142 \pm 0.039$ & 0.672 & -0.487 & -0.060 \\
\hline French_South & 0.675 & 0.195 & 0.13 & 0.636 & $0.589-0.738$ & 0.256 & $0.111-0.323$ & 0.108 & $0.088-0.151$ & $0.636 \pm 0.116$ & $0.225 \pm 0.165$ & $0.140 \pm 0.057$ & -0.003 & 0.189 & -0.558 \\
\hline Greek & 0.792 & 0.058 & 0.151 & 0.791 & $0.780-0.816$ & 0.048 & $0.019-0.060$ & 0.161 & $0.150-0.171$ & $0.658 \pm 0.098$ & $0.255 \pm 0.127$ & $0.086 \pm 0.039$ & 1.357 & -1.627 & 1.915 \\
\hline Hungarian & 0.558 & 0.264 & 0.179 & 0.548 & $0.520-0.590$ & 0.279 & $0.199-0.313$ & 0.174 & $0.156-0.210$ & $0.391 \pm 0.109$ & $0.454 \pm 0.153$ & $0.155 \pm 0.050$ & 1.437 & -1.145 & 0.371 \\
\hline Icelandic & 0.394 & 0.456 & 0.15 & 0.409 & $0.386-0.424$ & 0.448 & $0.409-0.473$ & 0.143 & $0.126-0.170$ & $0.342 \pm 0.102$ & $0.476 \pm 0.137$ & $0.182 \pm 0.045$ & 0.654 & -0.204 & -0.861 \\
\hline Lithuanian & 0.364 & 0.464 & 0.172 & 0.352 & $0.327-0.384$ & 0.488 & $0.433-0.527$ & 0.16 & $0.135-0.184$ & $0.248 \pm 0.117$ & $0.548 \pm 0.163$ & $0.205 \pm 0.052$ & 0.886 & -0.367 & -0.864 \\
\hline Maltese & 0.932 & 0 & 0.068 & & & & & & & $1.298 \pm 0.185$ & $-0.509 \pm 0.248$ & $0.211 \pm 0.079$ & & & \\
\hline Norwegian & 0.411 & 0.428 & 0.161 & 0.417 & $0.388-0.438$ & 0.423 & $0.383-0.450$ & 0.16 & $0.140-0.181$ & $0.273 \pm 0.115$ & $0.557 \pm 0.161$ & $0.170 \pm 0.055$ & 1.252 & -0.831 & -0.185 \\
\hline Orcadian & 0.457 & 0.385 & 0.158 & 0.465 & $0.439-0.493$ & 0.378 & $0.329-0.403$ & 0.157 & $0.140-0.179$ & $0.395 \pm 0.088$ & $0.437 \pm 0.122$ & $0.168 \pm 0.041$ & 0.798 & -0.487 & -0.264 \\
\hline Sardinian & 0.817 & 0.175 & 0.008 & 0.818 & $0.791-0.874$ & 0.141 & $0.058-0.182$ & 0.041 & $0.026-0.068$ & $0.883 \pm 0.128$ & $0.075 \pm 0.166$ & $0.042 \pm 0.048$ & -0.510 & 0.400 & -0.024 \\
\hline Scottish & 0.39 & 0.428 & 0.182 & 0.408 & $0.387-0.424$ & 0.421 & $0.384-0.448$ & 0.171 & $0.149-0.201$ & $0.286 \pm 0.112$ & $0.532 \pm 0.156$ & $0.182 \pm 0.053$ & 1.091 & -0.712 & -0.210 \\
\hline Sicilian & 0.903 & 0 & 0.097 & & & & & & & $1.012 \pm 0.149$ & $-0.131 \pm 0.199$ & $0.119 \pm 0.060$ & & & \\
\hline Spanish & 0.809 & 0.068 & 0.123 & 0.759 & $0.736-0.804$ & 0.126 & $0.066-0.170$ & 0.115 & $0.091-0.151$ & $0.856 \pm 0.126$ & $-0.015 \pm 0.165$ & $0.160 \pm 0.049$ & -0.769 & 0.855 & -0.922 \\
\hline Spanish_North & 0.713 & 0.125 & 0.163 & 0.612 & $0.561-0.660$ & 0.292 & $0.214-0.365$ & 0.096 & $0.072-0.126$ & $0.581 \pm 0.120$ & $0.298 \pm 0.158$ & $0.121 \pm 0.046$ & 0.254 & -0.038 & -0.533 \\
\hline Tuscan & 0.746 & 0.136 & 0.118 & 0.751 & $0.737-0.806$ & 0.123 & $0.047-0.145$ & 0.126 & $0.114-0.150$ & $0.734 \pm 0.118$ & $0.153 \pm 0.160$ & $0.113 \pm 0.054$ & 0.141 & -0.188 & 0.249 \\
\hline Ukrainian & 0.462 & 0.387 & 0.151 & 0.463 & $0.445-0.491$ & 0.376 & $0.322-0.399$ & 0.16 & $0.148-0.187$ & $0.259 \pm 0.123$ & $0.596 \pm 0.173$ & $0.145 \pm 0.057$ & 1.661 & -1.269 & 0.269 \\
\hline Finnish & & & & & & & & & & $-0.299 \pm 0.204$ & $1.194 \pm 0.296$ & $0.105 \pm 0.105$ & & & \\
\hline Mordovian & & & & & & & & & & $-0.255 \pm 0.173$ & $1.151 \pm 0.246$ & $0.104 \pm 0.090$ & & & \\
\hline Russian & & & & & & & & & & $-0.303 \pm 0.211$ & $1.230 \pm 0.301$ & $0.072 \pm 0.106$ & & & \\
\hline
\end{tabular}

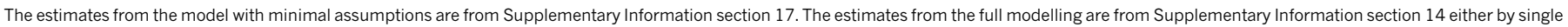
population analysis or co-fitting population pairs and averaging over fits (these averages are the results plotted in Fig. 4). Populations that do not fit the models are not reported. 